\title{
Optimal processing for seismic noise correlations
}

\author{
Andreas Fichtner ${ }^{1}$, Daniel Bowden ${ }^{1}$, and Laura Ermert ${ }^{2}$ \\ ${ }^{1}$ Department of Earth Sciences, ETH Zurich, Switzerland \\ ${ }^{2}$ Department of Earth and Planetary Sciences, Harvard University, U.S.A. \\ Corresponding author: Andreas Fichtner (andreas.fichtner@erdw.ethz.ch)
}

Submitted on 25 May 2020

\begin{abstract}
SUMMARY
A wide spectrum of processing schemes is commonly applied during the calculation of seismic noise correlations. This is intended to suppress large-amplitude transient and monochromatic signals, to accelerate convergence of the correlation process, or to modify raw correlations into more plausible approximations of inter-station Green's functions. Many processing schemes, such as one-bit normalisation or various nonlinear normalisations, clearly break the linear physics of seismic wave propagation. This naturally raises the question: To what extent are the resulting noise correlations physically meaningful quantities?

In this contribution, we demonstrate that commonly applied processing methods may indeed introduce an unphysical component into noise correlations. This affects noise correlation amplitudes but also, to a lesser extent, time-dependent phase information. The profound consequences are that most processed correlations cannot be entirely explained by any combination of Earth structure and noise sources, and that inversion results may thus be polluted.

The positive component of our analysis is a new and easily applicable method that allows us to modify any existing processing such that it becomes optimal in the sense of (1) completely avoiding the unphysical component, while (2) approximating the effects of the original processing as closely as possible. The resulting optimal schemes can be derived purely on the basis of observed noise, without any knowledge of or assumptions on the nature of noise sources.

In addition to the theoretical analysis, we present illustrative real-data examples from the Irish National Seismic Network and the Lost Hills array in Central California. We anticipate that optimal processing schemes may be most useful in applications that exploit complete correlation waveforms, amplitudes and low-amplitude arrivals, or small (time-dependent) phase shifts.
\end{abstract}

Key words: seismic noise, waveform analysis, seismic processing, seismic modelling, seismic waves

\section{INTRODUCTION}

Ambient noise interferometry rests on the extraction of deterministic information from the quasi-random ambient seismic field of the Earth. Most commonly, this is achieved via the correlation of noise from two stations, with the intent to approximate the inter-station Green's function (e.g. Lobkis \& Weaver, 2001; Shapiro \& Campillo, 2004; Weaver \& Lobkis, 2004; Wapenaar, 2004; Wapenaar \& Fokkema, 2006; Sánchez-Sesma \& Campillo, 2006; Weaver, 2008; Fichtner \& Tsai, 2019). Alternatives include interferometry by deconvolution (Snieder \& Şafak, 2006; Vasconcelos \& Snieder, 2008a,b; Snieder et al., 2009; van Dalen et al., 2014), phase cross-correlation (Schimmel et al., 2011), or multi-dimensional deconvolution (Wapenaar \& van der Neut, 2010; Wapenaar et al., 2011a,b). More recently, approaches to waveform interferometry have been developed in order to avoid interpreting the correlation as a Green's function, and instead directly interpret the waveform resulting from any distribution of noise sources (Tromp et al., 2010; Hanasoge, 2013; Delaney et al., 2017; Ermert et al., 2017; Sager et al., 2018b; Datta et al., 2019; Sager et al., 2020).

Regardless of the method used to extract coherent signals, noise interferometry is generally hampered by complexities of the seismic wavefield itself. The sources of the ambient field, mostly related to ocean wave activity, tend to be localised, mobile and transient (Ardhuin et al., 2011, 2015; Delaney et al., 2017; Gualtieri et al., 2019; Retailleau \& Gualtieri, 2019), thus leading to a non-diffuse wavefield to which 


\section{Andreas Fichtner, Daniel Bowden \& Laura Ermert}

arguments from diffuse acoustics from Green's function retrieval cannot be applied directly (Mulargia, 2012). Instead of being broadband, the ambient field tends to be dominated by few spectral peaks (e.g. Peterson, 1993; Stutzmann et al., 2012; McNamara \& Boaz, 2019). Furthermore, the Earth's natural seismicity or other high-energy events superimpose large-amplitude transient wavefields onto the generally low-amplitude ambient field.

The negative consequences of these complexities for ambient noise interferometry are numerous. Interferograms, e.g., inter-station correlations, may converge slowly towards a well-defined deterministic signal, thereby increasing the required amount of data and computation. The non-stationarity and heterogeneity of all combined wavefield sources may lead to poor Green's function approximations, including welldocumented biases in amplitudes and traveltimes, and the appearance of "spurious" arrivals (e.g. Halliday \& Curtis, 2008; Yao \& van der Hilst, 2009; Tsai, 2009; Froment et al., 2010; Tsai, 2011; Zhan et al., 2013; Fichtner, 2014, 2015; Yoritomo \& Weaver, 2016; Luo et al., 2020). Furthermore, the multitude of phenomena that affect an interferogram significantly complicates the solution of inverse problems, typically aiming to infer more isolated pieces of information, such as Earth structure from local to global scales (e.g. Shapiro et al., 2005; Stehly et al., 2009; Saygin \& Kennett, 2012; Poli et al., 2012; Haned et al., 2016; Bowden et al., 2016; Shapiro, 2019; Nakata \& Nishida, 2019) or ambient field sources (e.g. Stehly et al., 2006; Tian \& Ritzwoller, 2015; Ermert et al., 2016, 2017; Datta et al., 2019; Xu et al., 2019).

To circumvent these problems, a large variety of processing schemes have been developed. They include the averaging of causal and acausal correlation branches, spectral whitening, time-domain running averages, and frequency-domain normalisation (e.g. Bensen et al., 2007; Groos et al., 2012), one-bit normalisation (e.g. Larose et al., 2004; Shapiro \& Campillo, 2004; Cupillard et al., 2011; Hanasoge \& Branicki, 2013), phase-weighted stacks based on the Hilbert transform (Schimmel \& Paulssen, 1997; Schimmel et al., 2011) or the S transform (Baig et al., 2009), directional balancing (Curtis \& Halliday, 2010), temporal balancing (Weaver \& Yoritomo, 2018), Welch's method of overlapping time windows (Welch, 1967; Seats et al., 2012), the application of curvelet denoising filters (Stehly et al., 2011), sequences of selection and noise suppression filters (Nakata et al., 2015), frequency-time normalisation (Shen et al., 2012), or array-based processing using spectral analysis of the correlation matrix (e.g. Menon et al., 2012; Melo et al., 2013; Seydoux et al., 2017). A comprehensive overview of common processing schemes is provided by Ritzwoller \& Feng (2019).

Most of these processing schemes are nonlinear. Consequently, neither the processed recordings nor the processed interferograms actually satisfy the wave equation. This obvious fact is in contradiction to the common practice of interpreting processed interferograms as if they corresponded to a wavefield governed by a wave equation. Breaking the linear physics of wave propagation by nonlinear processing naturally raises questions about the extent to which processed interferograms may actually be unphysical.

Focusing on the most widely used interferometry by correlation, the present work has two main objectives. (1) To rigorously quantify the unphysical component in noise correlations induced by nonlinear processing, and (2) to develop new processing schemes that are optimal in the sense of being as close as possible to an existing nonlinear processing scheme, while completely avoiding the unphysical component. In this regard, our goal is not to develop the best processing scheme (in whatever sense), but to provide a tool to modify some preferred processing such that it becomes entirely physical and optimally close to its original purpose.

This manuscript is organised as follows. Based on the generalised interferometry concept of Fichtner et al. (2017), sections 2.1 and 2.2 develop explicit expressions for unprocessed and processed correlations. This directly leads, in sections 2.3 and 2.4 , to a quantification of the processing-induced unphysical component and to the desired optimal processing schemes. Finally, real-data examples at regional and local scales can be found in section 3 .

\section{THEORY}

In the interest of readability, we initially limit the following theoretical developments to the scalar, acoustic case. The conceptually identical but notationally more involved generalisation to the vectorical, elastic case can be found in Appendix B.

\subsection{Raw correlations and the interferometric wavefield}

For the calculation of raw (unprocessed) correlations, we begin with a displacement field $u\left(\mathbf{x}_{i}, t\right)$, observed at position $\mathbf{x}_{i}$ and within $N$ possibly overlapping time intervals, $t \in\left[t_{\text {start }}^{(n)}, t_{\text {end }}^{(n)}\right]$, for $n=1, \ldots, N$. In the frequency domain, the displacement field in the $n^{\text {th }}$ interval, $u^{(n)}\left(\mathbf{x}_{i}\right)$, is given by the representation theorem (e.g., Kennett, 2001; Aki \& Richards, 2002),

$u^{(n)}\left(\mathbf{x}_{i}\right)=\int G\left(\mathbf{x}_{i}, \mathbf{x}\right) F^{(n)}(\mathbf{x}) d \mathbf{x}+\varepsilon_{i}^{(n)}$,

where $G$ is the Green's function, $F^{(n)}$ is an external force, and $\varepsilon_{i}^{(n)}$ is noise sensu stricto, explained below. Both $F^{(n)}$ and $\varepsilon_{i}^{(n)}$ depend on the time interval $n$. The integral in (1) is over the entire volume of the Earth, and the length of the time interval is assumed to be much longer than the longest significant period in $F^{(n)}$. Since we continue to work in the frequency domain, we do not introduce special symbols for frequency-domain quantities, and for notational simplicity we omit dependencies on the circular frequency $\omega$. 
The Green's function $G$ contains the complete linear response of the Earth (including its solid, fluid and gaseous components) to any kind of force $F^{(n)}$ that is small enough to justify linearity. Hence, $G$ includes deformation due to tidal forces, atmospheric pressure variations, ambient and cultural noise sources, but also static and transient displacement outside the source region of earthquakes or explosions. In this sense, the noise $\varepsilon_{i}^{(n)}$ is that part of the observations $u^{(n)}\left(\mathbf{x}_{i}\right)$ that cannot be captured by a linear forward modelling theory. In most practical applications, $\varepsilon_{i}^{(n)}$ is incoherent instrumental noise.

Using (1), the correlation function or interferogram between two receivers $\mathbf{x}_{i}$ and $\mathbf{x}_{k}$ in time interval $n$ is

$I^{(n)}\left(\mathbf{x}_{i}, \mathbf{x}_{k}\right)=u^{(n)}\left(\mathbf{x}_{i}\right) u^{(n) *}\left(\mathbf{x}_{k}\right)=\iint G\left(\mathbf{x}_{i}, \mathbf{x}\right) G^{*}\left(\mathbf{x}_{k}, \mathbf{x}^{\prime}\right) S^{(n)}\left(\mathbf{x}, \mathbf{x}^{\prime}\right) d \mathbf{x} d \mathbf{x}^{\prime}+E^{(n)}\left(\mathbf{x}_{i}, \mathbf{x}_{k}\right)$,

with the source power-spectral density $S^{(n)}\left(\mathbf{x}, \mathbf{x}^{\prime}\right)=F^{(n)}(\mathbf{x}) F^{(n) *}\left(\mathbf{x}^{\prime}\right)$. The asterisk denotes complex conjugation, and the term $E^{(n)}\left(\mathbf{x}_{i}, \mathbf{x}_{k}\right)$ contains all contributions to $I^{(n)}\left(\mathbf{x}_{i}, \mathbf{x}_{i}\right)$ that involve $\varepsilon_{i}^{(n)}$ or $\varepsilon_{k}^{(n)}$. To obtain a stable and largely deterministic interferogram, we average over all time intervals to obtain the ensemble interferogram

$I\left(\mathbf{x}_{i}, \mathbf{x}_{k}\right)=\frac{1}{N} \sum_{n=1}^{N} I^{(n)}\left(\mathbf{x}_{i}, \mathbf{x}_{k}\right)=\iint G\left(\mathbf{x}_{i}, \mathbf{x}\right) G^{*}\left(\mathbf{x}_{k}, \mathbf{x}^{\prime}\right) S\left(\mathbf{x}, \mathbf{x}^{\prime}\right) d \mathbf{x} d \mathbf{x}^{\prime}+\frac{1}{N} \sum_{n=1}^{N} E^{(n)}\left(\mathbf{x}_{i}, \mathbf{x}_{k}\right)$,

with the average source power-spectral density $S\left(\mathbf{x}, \mathbf{x}^{\prime}\right)=\sum_{n=1}^{N} S^{(n)}\left(\mathbf{x}, \mathbf{x}^{\prime}\right) / N$. For a sufficiently large number $N$ of time intervals, we expect $\sum_{n=1}^{N} E^{(n)}\left(\mathbf{x}_{i}, \mathbf{x}_{k}\right) / N$ in (3) to become negligibly small. By changing the order of integration, Eq. (3) can be rearranged into a representation theorem, whereby a wavefield is written in terms of an integral over a Green's function times a source term,

$I\left(\mathbf{x}_{i}, \mathbf{x}_{k}\right)=\underbrace{\int G\left(\mathbf{x}_{i}, \mathbf{x}\right) \underbrace{\left[\int G^{*}\left(\mathbf{x}_{k}, \mathbf{x}^{\prime}\right) S\left(\mathbf{x}, \mathbf{x}^{\prime}\right) d \mathbf{x}^{\prime}\right]}_{\text {source }} d \mathbf{x}}_{\text {interferometric wavefield }}+\underbrace{\frac{1}{N} \sum_{n=1}^{N} E^{(n)}\left(\mathbf{x}_{i}, \mathbf{x}_{k}\right)}_{\text {noise senu stricto }}$.

Eq. (4) implies that the correlation $I\left(\mathbf{x}_{i}, \mathbf{x}_{k}\right)$ is equivalent to an interferometric wavefield observed at variable positions $\mathbf{x}_{i}$ and for a fixed reference station $\mathbf{x}_{k}$ (see also Lin et al., 2013; Fichtner, 2014). It is excited by the source term in square brackets, and it is propagated through the medium towards position $\mathbf{x}_{i}$ via the Green's function $G\left(\mathbf{x}_{i}, \mathbf{x}\right)$. The interpretation of $I\left(\mathbf{x}_{i}, \mathbf{x}_{k}\right)$ as a propagating wavefield is central for the following developments, where we will continue to consider $\mathbf{x}_{i}$ variable and $\mathbf{x}_{k}$ fixed.

The interpretation of $I\left(\mathbf{x}_{i}, \mathbf{x}_{k}\right)$ also provides an efficient algorithm for the computation of synthetic correlations: For a suitable Earth model, we first compute the Green's function $G\left(\mathbf{x}_{k}, \mathbf{x}^{\prime}\right)$ with source at the reference station $\mathbf{x}_{k}$. The integral $\int G^{*}\left(\mathbf{x}_{k}, \mathbf{x}^{\prime}\right) S\left(\mathbf{x}, \mathbf{x}^{\prime}\right) d \mathbf{x}^{\prime}$ then provides the source term for the computation of the interferometric wavefield, possibly using the same solver as for the computation of $G\left(\mathbf{x}_{k}, \mathbf{x}^{\prime}\right)$. In the interest of numerical efficiency, the source power-spectral density is in most practical applications assumed to have the form $S\left(\mathbf{x}, \mathbf{x}^{\prime}\right)=S(\mathbf{x}) \delta\left(\mathbf{x}-\mathbf{x}^{\prime}\right)$, which eliminates one of the integrals in (4) (e.g. Ermert et al., 2017; Datta et al., 2019; Sager et al., 2020). For the developments presented here, this simplification is, however, not necessary.

\subsection{Processed correlations and effective physics}

Processing usually aims to make the wavefield more stationary and to reduce the bias caused by stronger sources in time or space. For this, various techniques have been invented which act on each signal, i.e., waveforms for a station pair and a time window, independently. These include, for instance, one-bit normalisation, causal-acausal averaging, and many others. As we will shortly demonstrate, however, processing that can be interpreted as having a physically meaningful result is more restrictive. We desire only processing that equally affects all station pairs $(i, k)$ in a given time window $n$, or conversely that affects a given station pair $(i, k)$ equally across all time windows $n$. Any existing or new processing scheme, $T$, can be shown to have some combination of these two components, plus some additional component of nonphysical effects that we wish to identify and eliminate. This distinction between time-window- and station-pair-specific processing motivates a factorisation of $T$.

Processing transforms the raw interferogram $I^{(n)}\left(\mathbf{x}_{i}, \mathbf{x}_{k}\right)$ into the processed interferogram

$\tilde{I}^{(n)}\left(\mathbf{x}_{i}, \mathbf{x}_{k}\right)=T\left[I^{(n)}\left(\mathbf{x}_{i}, \mathbf{x}_{k}\right)\right]$

where $T$ is a transfer function. Often, the transfer function cannot be written in a simple analytical form. Instead, it may be described in the form of a computational recipe.

For many processing schemes, $T$ is nonlinear in the sense of breaking standard linear wave propagation physics. For example, the application of a bandpass filter is by itself a linear processing that can be applied to the complete wave equations, and which is therefore ultimately equivalent to the action of a bandpass filtered source. However, the application of two different bandpasses to recordings at two different stations is a nonlinear processing, because it cannot be represented by a bandpass filtered source that radiates a bandpass filtered wavefield.

Since any dataset contains only a finite number of interferograms $I^{(n)}\left(\mathbf{x}_{i}, \mathbf{x}_{k}\right)$, we can limit ourselves to evaluations of $T$ at discrete points. 
a) Transfer function $T$

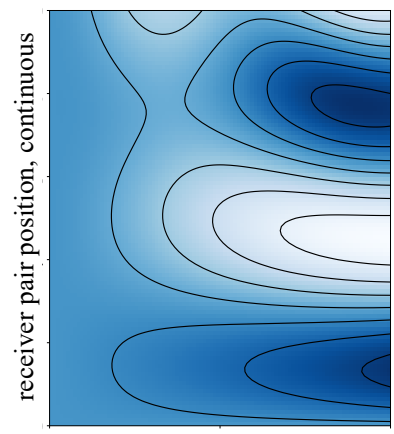

window, continuous b) Transfer coefficients $T_{i k}^{(n)}$

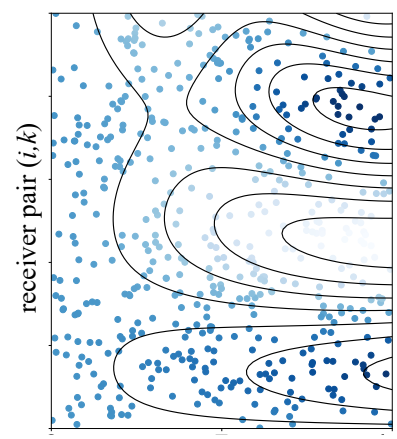

window $n$ c) Factorisation $f^{(n)} g_{i k}$

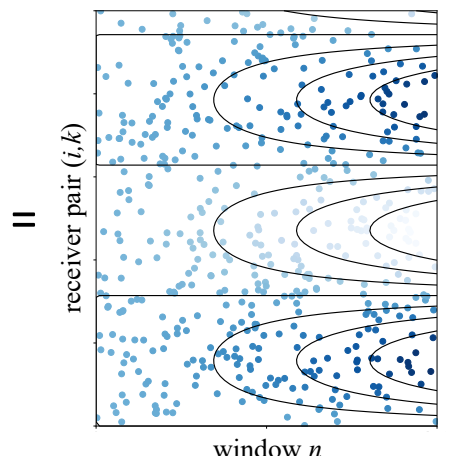

d) Residual $e_{i k}^{(n)}$

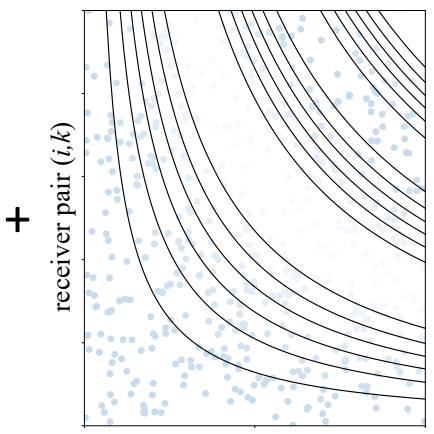

window $n$

Figure 1. Schematic illustration of the transfer function and its discrete factorisation. (a) The transfer function $T$, introduced in Eq. (5) is a continuous and potentially highly nonlinear function that transforms an unprocessed (raw) interferogram into its processed version. It is illustrated here as an arbitrary function of the time window and the receiver pair position. (b) The transfer coefficient $T_{i k}^{(n)}$ is a point-wise discretisation of the originally continuous transfer function. The points, labelled by window index $n$ and receiver pair index $(i, k)$, are the recordings in a specific dataset of finite size. The recordings are shown schematically in the form of coloured points. (c) Following Eq. (8), the transfer coefficient $T_{i k}^{(n)}$ can be factorised into $f^{(n)} g_{i k}$. As a consequence, processing along the receiver-pair axis becomes independent of the time window, and processing on the time window axis becomes independent of the receiver pair. (d) The factorisation of $T_{i k}^{(n)}$ into $f^{(n)} g_{i k}$ is generally not perfect, and a residual $e_{i k}^{(n)}$ is left. It corresponds to processing for a receiver pair $(i, k)$ that is different for each time window $n$, and cannot be made independent of it.

For this, we rearrange (5) into

$\tilde{I}^{(n)}\left(\mathbf{x}_{i}, \mathbf{x}_{k}\right)=\frac{T\left[I^{(n)}\left(\mathbf{x}_{i}, \mathbf{x}_{k}\right)\right]}{I^{(n)}\left(\mathbf{x}_{i}, \mathbf{x}_{k}\right)} I^{(n)}\left(\mathbf{x}_{i}, \mathbf{x}_{k}\right)=T_{i k}^{(n)} I^{(n)}\left(\mathbf{x}_{i}, \mathbf{x}_{k}\right)$,

with the transfer coefficient $T_{i k}^{(n)}$. Eq. (6) constitutes a discretisation of the continuous transfer function $T$ at a finite number of grid points in interferogram space, indexed by $n, i$, and $k$. The discretisation is schematically illustrated in Fig. 1a,b.

A major benefit of the discretisation (6) is that a closed analytic expression of the potentially complicated transfer function becomes unnecessary. In fact, we may compute the transfer coefficients $T_{i k}^{(n)}$ by simple spectral division of the raw and processed interferograms, that is,

$T_{i k}^{(n)}=\frac{\tilde{I}^{(n)}\left(\mathbf{x}_{i}, \mathbf{x}_{k}\right)}{I^{(n)}\left(\mathbf{x}_{i}, \mathbf{x}_{k}\right)}=\frac{\tilde{I}^{(n)}\left(\mathbf{x}_{i}, \mathbf{x}_{k}\right) I^{(n) *}\left(\mathbf{x}_{i}, \mathbf{x}_{k}\right)}{\left|I^{(n)}\left(\mathbf{x}_{i}, \mathbf{x}_{k}\right)\right|^{2}}$.

Eq. (7) yields the transfer coefficient $T_{i k}^{(n)}$ for frequencies where the spectral power of the raw interferogram, $\left|I^{(n)}\left(\mathbf{x}_{i}, \mathbf{x}_{k}\right)\right|$, is sufficiently large to produce a numerically stable division.

Processing often removes or corrects for effects that are either specific to a time window $n$ or a receiver pair $(i, k)$. For instance, time windows $n$ containing high levels of anthropogenic noise with very localised sources may be down-weighted relative to time windows where ambient noise dominates. As another example, receiver-pair-specific processing includes averaging of the causal and anti-causal parts of a correlation function. A receiver pair $(i, k)$ that is favourably oriented with respect to ambient noise sources may be nearly symmetric, meaning that $T_{i k}^{(n)} \approx 1$. In contrast, a differently oriented receiver pair $\left(i^{\prime}, k^{\prime}\right)$ may be strongly asymmetric, resulting in a transfer coefficient $T_{i^{\prime} k^{\prime}}^{(n)} \neq 1$. The intuitive distinction between time-window- and receiver-pair-specific processing motivates a factorisation of the transfer coefficient, $T_{i k}^{(n)}=f^{(n)} g_{i k}+e_{i k}^{(n)}$.

The factor $f^{(n)}$ represents processing applied to time window $n$, irrespective of the receiver pair $(i, k)$. As this term collects / represents the component of processing that affects all station pairs equally, we can ensure wave propagation physics are spatially preserved. Analogously, $g_{i k}$ is the component of processing applied to receiver pair $(i, k)$ for any time window $n$. This term effectively mandates that a given receiver pair is processed consistently for all time windows, and thus ensures that a changing distribution of noise sources will not break linearity and favour one station pair over any other. The factorisation residual $e_{i k}^{(n)}$ captures the remaining processing that is neither only time-windownor receiver-pair-specific. A schematic illustration of the factorisation is shown in Fig. 1c,d. In practice, this factorisation is straightforward to find. It is detailed further in Appendices A and B, and a step-by-step example is given in section 3.1.

Clearly, there are infinitely many specific realisations of the factorisation (8). However, as we will see in section 2.3, the minimisation of processing-induced unphysical effects requires us to find $f^{(n)}$ and $g_{i k}$ such that the residual $e_{i k}^{(n)}$ is as small as possible. 
Eq. (8) allows us to represent the processed interferograms $\tilde{I}^{(n)}\left(\mathbf{x}_{i}, \mathbf{x}_{k}\right)$ on the basis of Eq. (2) as

$$
\begin{aligned}
\tilde{I}^{(n)}\left(\mathbf{x}_{i}, \mathbf{x}_{k}\right) & =\int g_{i k} G\left(\mathbf{x}_{i}, \mathbf{x}\right)\left[\int G^{*}\left(\mathbf{x}_{k}, \mathbf{x}^{\prime}\right) f^{(n)} S^{(n)}\left(\mathbf{x}, \mathbf{x}^{\prime}\right) d \mathbf{x}^{\prime}\right] d \mathbf{x}+\tilde{E}^{(n)}\left(\mathbf{x}_{i}, \mathbf{x}_{k}\right) \\
& +\int G\left(\mathbf{x}_{i}, \mathbf{x}\right)\left[\int G^{*}\left(\mathbf{x}_{k}, \mathbf{x}^{\prime}\right) e_{i k}^{(n)} S^{(n)}\left(\mathbf{x}, \mathbf{x}^{\prime}\right) d \mathbf{x}^{\prime}\right] d \mathbf{x} .
\end{aligned}
$$

The term $\tilde{E}^{(n)}\left(\mathbf{x}_{i}, \mathbf{x}_{k}\right)$ in (9) represents the processing $T_{i k}^{(n)}$ applied to the noise term $E^{(n)}\left(\mathbf{x}_{i}, \mathbf{x}_{k}\right)$. Again averaging over all time intervals, we obtain the processed ensemble interferogram

$$
\begin{aligned}
\tilde{I}\left(\mathbf{x}_{i}, \mathbf{x}_{k}\right) & =\underbrace{\int g_{i k} G\left(\mathbf{x}_{i}, \mathbf{x}\right) \underbrace{\left[\int G^{*}\left(\mathbf{x}_{k}, \mathbf{x}^{\prime}\right) \tilde{S}\left(\mathbf{x}, \mathbf{x}^{\prime}\right) d \mathbf{x}^{\prime}\right]}_{\text {effective source }} d \mathbf{x}}_{\text {processed interferometric wavefield }}+\underbrace{\frac{1}{N} \sum_{n=1}^{N} \tilde{E}^{(n)}\left(\mathbf{x}_{i}, \mathbf{x}_{k}\right)}_{\text {proc. noise sensu stricto }} \\
& +\underbrace{\int G\left(\mathbf{x}_{i}, \mathbf{x}\right) \underbrace{\left[\int G^{*}\left(\mathbf{x}_{k}, \mathbf{x}^{\prime}\right) \tilde{S}_{i k}\left(\mathbf{x}, \mathbf{x}^{\prime}\right) d \mathbf{x}^{\prime}\right]}_{\text {unhysical wavefield source }} d}_{\text {unphysical wavefield }},
\end{aligned}
$$

with the effective source power-spectral densities

$\tilde{S}\left(\mathbf{x}, \mathbf{x}^{\prime}\right)=\frac{1}{N} \sum_{n=1}^{N} f^{(n)} S^{(n)}\left(\mathbf{x}, \mathbf{x}^{\prime}\right), \quad \tilde{S}_{i k}\left(\mathbf{x}, \mathbf{x}^{\prime}\right)=\frac{1}{N} \sum_{n=1}^{N} e_{i k}^{(n)} S^{(n)}\left(\mathbf{x}, \mathbf{x}^{\prime}\right)$.

Comparing (4) and (10) demonstrates that processing has multiple effects. The original power-spectral density of the sources, $S$, is transformed into an effective power-spectral density, $\tilde{S}$. Consequently, the interferometric wavefield is excited by a new effective source. In this context, the factor $f^{(n)}$ from (8) acquires the meaning of a source corrector. Furthermore, the interferometric wavefield propagates through the medium via an effective Green's function, $g_{i k} G\left(\mathbf{x}_{i}, \mathbf{x}\right)$. The effective Green's function differs from the original Green's function $G\left(\mathbf{x}_{i}, \mathbf{x}\right)$ by the frequency-dependent propagation corrector $g_{i k}$. Similar to (4), the term $\sum_{n=1}^{N} \tilde{E}^{(n)}\left(\mathbf{x}_{i}, \mathbf{x}_{k}\right) / N$ represents the contribution of noise sensu stricto, which we expect to become negligibly small as $N$ increases.

\subsection{The unphysical wavefield}

Eqs. (4) and (10) also differ in the last term on the right-hand side, involving the effective power-spectral density $\tilde{S}_{i k}$. In fact, the integral $\int G^{*}\left(\mathbf{x}_{k}, \mathbf{x}^{\prime}\right) \tilde{S}_{i k}\left(\mathbf{x}, \mathbf{x}^{\prime}\right) d \mathbf{x}^{\prime}$ represents a wavefield source that depends on the observation point $\mathbf{x}_{i}$. Therefore, observers at different locations effectively 'see' wavefields from different sources, as illustrated in Fig. 2. In this sense, the term in (10) involving $\tilde{S}_{i k}$ is inconsistent with classical wave propagation physics.

The presence of the unphysical wavefield implies that processed correlations cannot generally be interpreted as true physical correlations excited by a unique (effective) source and propagated through a unique (effective) medium. Thus, observers of processed correlations, used to our normal physical reality, must encounter discrepancies, which they may erroneously map into spurious variations in Earth structure.

An unpleasant computational consequence of the unphysical wavefield is that processed interferograms for all possible observation points $\mathbf{x}_{i}$ cannot be computed using only the universal wavefield source $\int G^{*}\left(\mathbf{x}_{k}, \mathbf{x}^{\prime}\right) \tilde{S}\left(\mathbf{x}, \mathbf{x}^{\prime}\right) d \mathbf{x}^{\prime}$. Instead, a different source $\int G^{*}\left(\mathbf{x}_{k}, \mathbf{x}^{\prime}\right) \tilde{S}_{i k}\left(\mathbf{x}, \mathbf{x}^{\prime}\right)$ must be used for each of the possible observation points $\mathbf{x}_{i}$, which drastically increases the required computational resources. (The same is not true for $g_{i k}$, which can always be pulled out of the integral.)

The amplitude of the unphysical wavefield in (10) depends linearly on the factorisation residual $e_{i k}^{(n)}$, which is, $a$ priori, not uniquely determined from the definition of the transfer coefficient factorisation (8). To minimise both the unphysical wavefield and the computational forward modelling requirements, it is desirable to determine the factors such that most of the processing effect is captured by effective wave propagation physics and sources, while minimising the unphysical component related to $e_{i k}^{(n)}$. The solution of this minimisation problem is presented in Appendix A.

\subsection{Optimal processing}

We define a processing to be optimal when the resulting unphysical wavefield vanishes exactly. The goal of designing an optimal processing that mimics a traditional (usually non-optimal) processing as closely as possible can be reached as follows: First, we compute raw and processed interferograms, $I^{(n)}\left(\mathbf{x}_{i}, \mathbf{x}_{k}\right)$ and $\tilde{I}^{(n)}\left(\mathbf{x}_{i}, \mathbf{x}_{k}\right)$ for each time interval. From this we obtain the factors $f^{(n)}, g_{i k}$ and $e_{i k}^{(n)}$ of the transfer coefficients $T_{i k}^{(n)}$. Since the factorisation residual $e_{i k}^{(n)}$ is optimally small, the effect of the processing will be captured as much as possible by the propagation corrector $g_{i k}$ and the source corrector $f^{(n)}$. Subsequently, we compute optimally processed interferograms per 


\section{Andreas Fichtner, Daniel Bowden \& Laura Ermert}

a) regular wave propagation

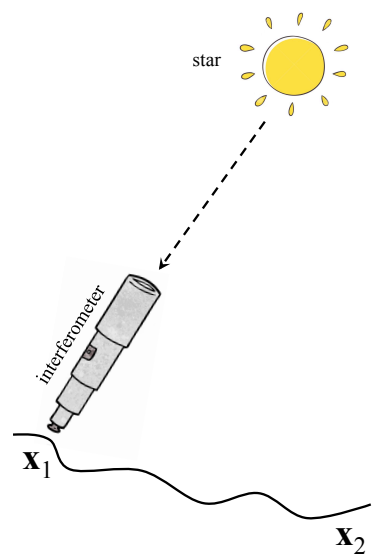

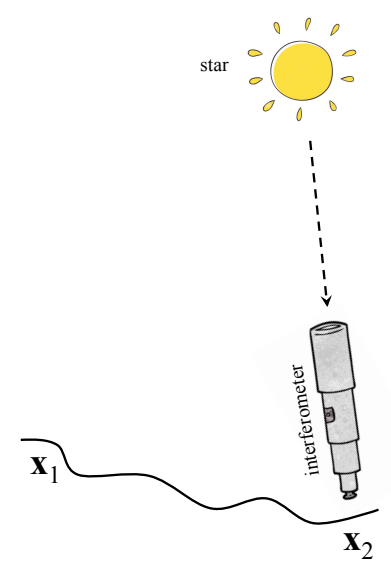

b) unphysical wave propagation

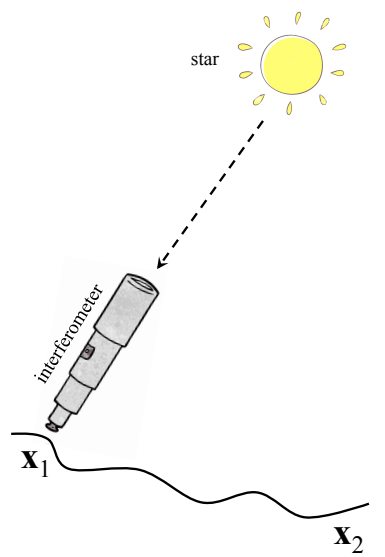

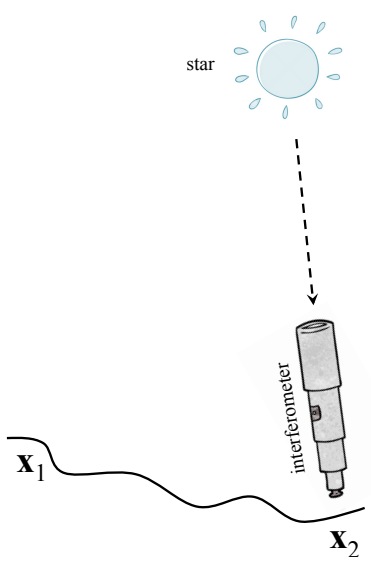

Figure 2. Illustration of the unphysical wavefield effect. (a) A distant star is observed from two different positions, $\mathbf{x}_{1}$ and $\mathbf{x}_{2}$, using an interferometer. The same object (the wavefield source, coloured yellow) is observed from both positions, according to classical wave propagation physics. (b) Processing of the incoming wavefield make the star appear brighter at position $\mathbf{x}_{1}$, which corresponds to a subjectively improved noise correlation function. However, it also causes the observer at position $\mathbf{x}_{2}$ to see a different source (coloured blue) than the observer at position $\mathbf{x}_{1}$. Not knowing about the processing effect, the observer at $\mathbf{x}_{2}$ must conclude that the atmosphere between the star and the interferometer must be different than at location $\mathbf{x}_{1}$, that is, the difference in the observation is incorrectly ascribed to the medium, while in reality it is a modification of the source appearance by processing.

time interval as

$\tilde{I}_{\mathrm{opt}}^{(n)}\left(\mathbf{x}_{i}, \mathbf{x}_{k}\right)=f^{(n)} g_{i k} I^{(n)}\left(\mathbf{x}_{i}, \mathbf{x}_{k}\right)$.

Thus, optimal processing is accomplished through the multiplication of the raw interferogram with the propagation corrector and the source corrector, explicitly omitting the factorisation residual $e_{i k}^{(n)}$. Using (2), we can express $\tilde{I}_{\mathrm{opt}}^{(n)}\left(\mathbf{x}_{i}, \mathbf{x}_{k}\right)$ as

$\tilde{I}_{\mathrm{opt}}^{(n)}\left(\mathbf{x}_{i}, \mathbf{x}_{k}\right)=\int g_{i k} G\left(\mathbf{x}_{i}, \mathbf{x}\right)\left[\int G^{*}\left(\mathbf{x}_{k}, \mathbf{x}^{\prime}\right) f^{(n)} S^{(n)}\left(\mathbf{x}, \mathbf{x}^{\prime}\right) d \mathbf{x}^{\prime}\right] d \mathbf{x}+f^{(n)} g_{i k} E^{(n)}\left(\mathbf{x}_{i}, \mathbf{x}_{k}\right)$.

Averaging over all time intervals, yields the optimally processed ensemble interferogram

$\tilde{I}_{\mathrm{opt}}\left(\mathbf{x}_{i}, \mathbf{x}_{k}\right)=\underbrace{\int g_{i k} G\left(\mathbf{x}_{i}, \mathbf{x}\right)\left[\int G^{*}\left(\mathbf{x}_{k}, \mathbf{x}^{\prime}\right) \tilde{S}\left(\mathbf{x}, \mathbf{x}^{\prime}\right) d \mathbf{x}^{\prime}\right] d \mathbf{x}}_{\text {processed interferometric wavefield }}+\underbrace{\frac{1}{N} \sum_{n=0}^{N} f^{(n)} g_{i k} E^{(n)}\left(\mathbf{x}_{i}, \mathbf{x}_{k}\right)}_{\text {noise sensu stricto }}$.

By construction, the optimally processed interferogram is not contaminated by an unphysical wavefield. It can thus be correctly interpreted in terms of a wavefield excited by a unique effective source, and propagating through a unique effective medium.

The optimal processing scheme is not optimal in the sense of being the best of all possible processings. Instead, it is a modification of an existing processing scheme that has been found suitable for a specific dataset or class of applications. The modification ensures that the new processing is physical and optimally close to the original processing.

\subsection{Empirical estimation of the unphysical wavefield}

The precise computation of the unphysical wavefield based on (10) and (11) requires knowledge of the source power-spectral density $S^{(n)}\left(\mathbf{x}, \mathbf{x}^{\prime}\right)$ per time interval. Though this information is mostly unavailable, the unphysical wavefield may still be estimated empirically. For this, we subtract the optimally processed interferogram $\tilde{I}_{\mathrm{opt}}\left(\mathbf{x}_{i}, \mathbf{x}_{k}\right)$ from the non-optimally processed interferogram $\tilde{I}\left(\mathbf{x}_{i}, \mathbf{x}_{k}\right)$. Using (10) and (13), we find

$$
\tilde{I}\left(\mathbf{x}_{i}, \mathbf{x}_{k}\right)-\tilde{I}_{\mathrm{opt}}\left(\mathbf{x}_{i}, \mathbf{x}_{k}\right)=\underbrace{\int G\left(\mathbf{x}_{i}, \mathbf{x}\right)\left[\int G^{*}\left(\mathbf{x}_{k}, \mathbf{x}^{\prime}\right) \tilde{S}_{i k}\left(\mathbf{x}, \mathbf{x}^{\prime}\right) d \mathbf{x}^{\prime}\right] d \mathbf{x}}_{\text {unphysical wavefield }}+\frac{1}{N} \sum_{n=1}^{N} e_{i k}^{(n)} E^{(n)}\left(\mathbf{x}_{i}, \mathbf{x}_{k}\right) .
$$

The last term in (15) is proportional to products of the small quantities $e_{i k}^{(n)}, \varepsilon_{i}^{(n)}$ and $\varepsilon_{k}^{(n)}$, suggesting the following empirical approximation of the unphysical wavefield,

$\tilde{I}\left(\mathbf{x}_{i}, \mathbf{x}_{k}\right)-\tilde{I}_{\mathrm{opt}}\left(\mathbf{x}_{i}, \mathbf{x}_{k}\right) \approx \int G\left(\mathbf{x}_{i}, \mathbf{x}\right)\left[\int G^{*}\left(\mathbf{x}_{k}, \mathbf{x}^{\prime}\right) \tilde{S}_{i k}\left(\mathbf{x}, \mathbf{x}^{\prime}\right) d \mathbf{x}^{\prime}\right] d \mathbf{x}$

Eq. (16) is the basis of the unphysical wavefield estimates in the following real-data examples. 
a) Irish Seismic Network (ISN) stations

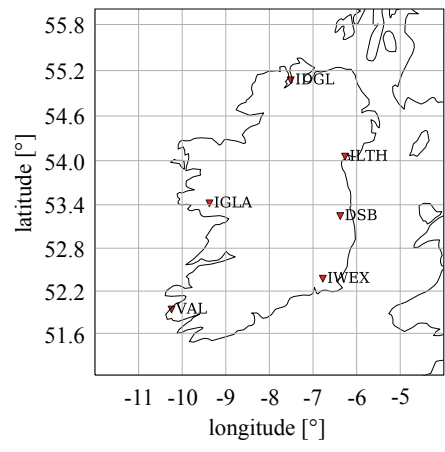

b) Station DSB, 1 January 2019
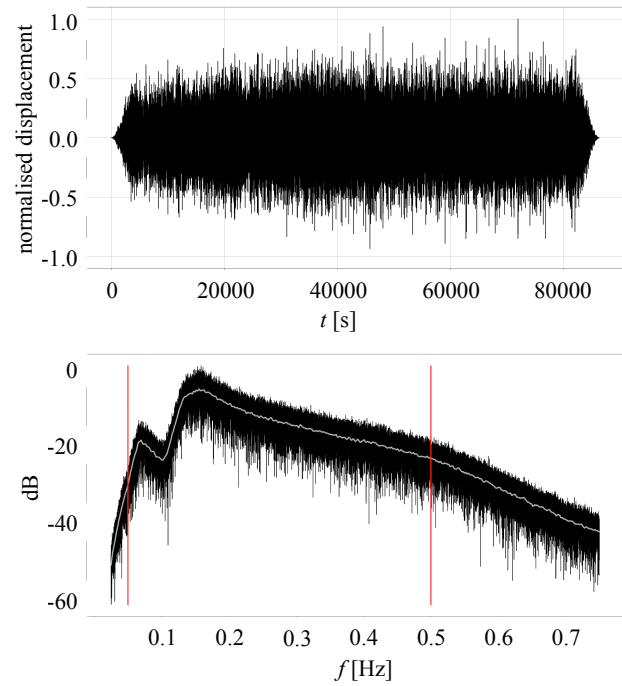

c) Station DSB, 5 January 2019
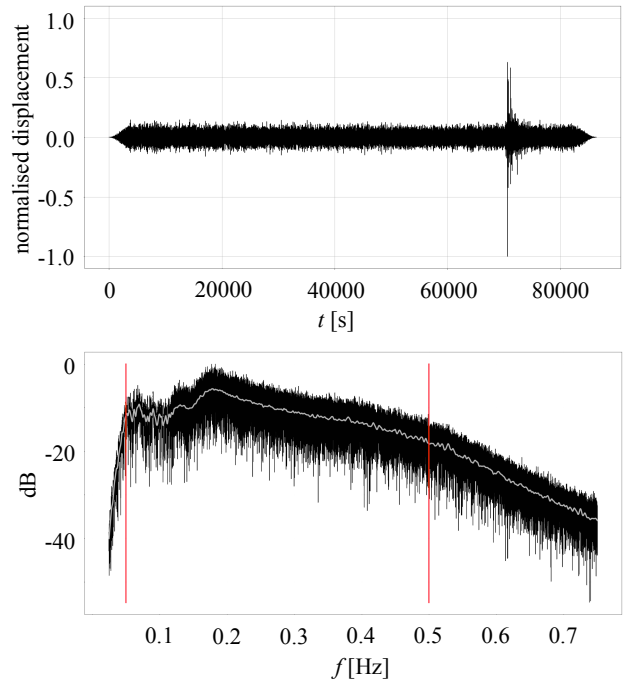

Figure 3. One-day-long vertical-component recordings on January 1 and 5, 2019, at station DSB, which is part of the Irish Seismic Network, shown in panel (a). Time-domain plots (top) display normalised displacement, and the frequency-domain plots (bottom) show normalised spectral amplitude. The grey line is a smoothed version of the spectrum, included for better visibility. The red vertical lines mark the boundaries of the frequency spectrum, used in the analyses below.

\section{EXAMPLES}

To illustrate the previous theoretical developments, we consider two real-data examples; a regional one with recordings from the Irish Seismic Network, and a local one with data from the Lost Hills array in Southern California (Clayton, 2015). The emphasis in these examples is on surface waves, as these are most widely used in ambient noise tomography. More detailed analyses, for instance, of body and coda waves will be the subject of future studies.

In contrast to the theory presented in section 2, the practical examples are based on discrete and not on continuous Fourier transforms. This has, however, no effect on the essence of the theory.

\subsection{Regional scale}

We first illustrate the theoretical developments from section 2 using a small regional-scale dataset from the Irish Seismic Network, which is summarised in Fig. 3. The dataset consists of vertical-component one-day-long recordings from 1 to 31 January 2019 at six stations located along the Irish coast. As expected on North Atlantic winter days, the majority of the recordings is dominated by microseismic noise with pronounced spectral peaks around $0.08 \mathrm{~Hz}$ and $0.17 \mathrm{~Hz}$. A typical example is shown in Fig. 3b. On some days, the recordings include largeamplitude transient events, such as the clearly visible surface-wave arrival from a deep M6.8 earthquake in NW Brazil, displayed in Fig. $3 \mathrm{c}$. These overall characteristics of the data suggest a combination of (1) spectral whitening to broaden the exploitable frequency range and (2) one-bit normalisation to automatically remove large-amplitude transients. Since processing for ambient noise interferometry is to a large extent subjective, other choices may well be justified.

Using Eq. (6), we compute transfer coefficients $T_{i k}^{(n)}$ by spectral division of raw and processed interferograms. The factorisation (8), detailed in Appendix A, then yields the source correctors $f^{(n)}$, the propagation correctors $g_{i k}$, and the factorisation residuals $e_{i k}^{(n)}$.

A representative selection of 6 out of 31 source correctors is shown in Fig. 4. Each of the source correctors modifies the temporal spectrum of the original source power-spectral densities $S^{(n)}$ to produce effective versions $\tilde{S}^{(n)}=f^{(n)} S^{(n)}$. This effect is independent of the receiver pair $(i, k)$, and it assigns relative weights to the sources acting in the different day-long time windows indexed by $n$. While there is strong variability among the source correctors, most of them resemble those on January 1 (Fig. 4a) and January 13 (Fig. 4d). A notable exception is, for instance, the source corrector on January 10 (Fig. 4c), which gives a significantly higher weight to that day, especially below $0.15 \mathrm{~Hz}$ and above $0.3 \mathrm{~Hz}$. By construction, the average of the source correctors over all windows (days from 1 to 31 January) is exactly 1 for all frequencies.

In contrast to the source correctors $f^{(n)}$, the propagation correctors $g_{i k}$ are receiver-pair- or path-specific. They describe the processinginduced modification from original to effective wave propagation physics via changes in amplitude and phase with which different frequency components of the wavefield are transported between $\mathbf{x}_{i}$ and $\mathbf{x}_{k}$. This modification is time-invariant, that is, independent of the time window 


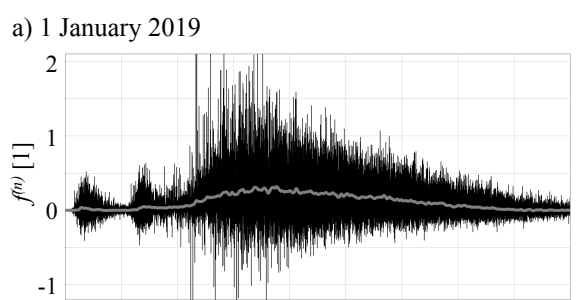

d) 13 January 2019

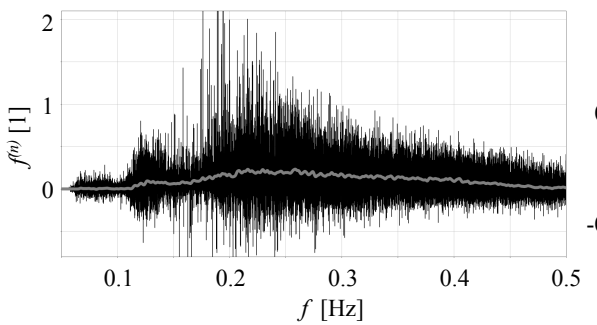

b) 4 January 2019

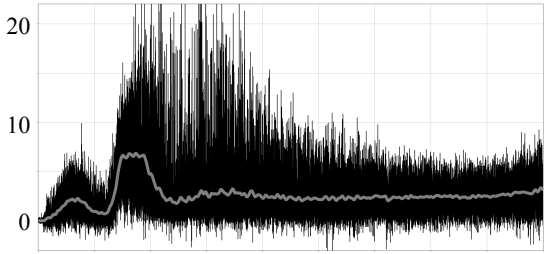

e) 22 January 2019

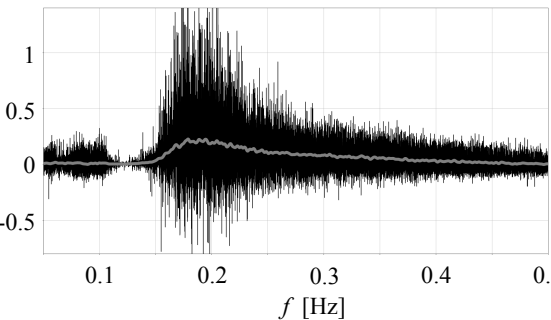

c) 10 January 2019

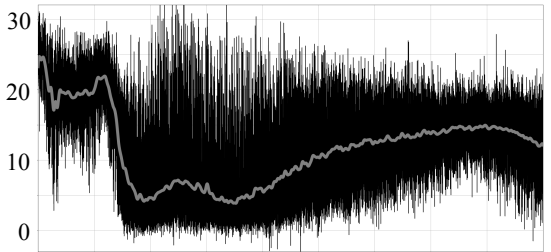

e) 26 January 2019

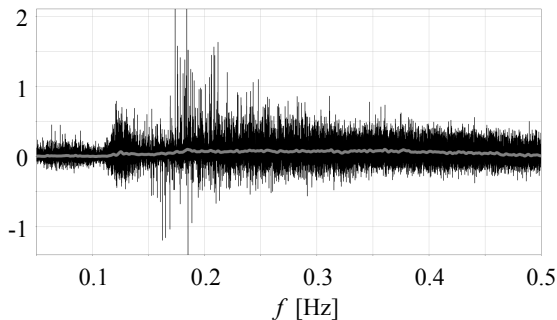

Figure 4. Selection of frequency-domain source correctors $f^{(n)}$ for six days in January 2019. The grey lines are smoothed versions of the spectra. Though the examples shown here have considerable variability, most source correctors are similar to those from January 1 (panel (a)) and January 13 (panel (d)). As shown in Appendix A, the source corrector is a real-valued quantity, which is why $f^{(n)}$ itself can be displayed.
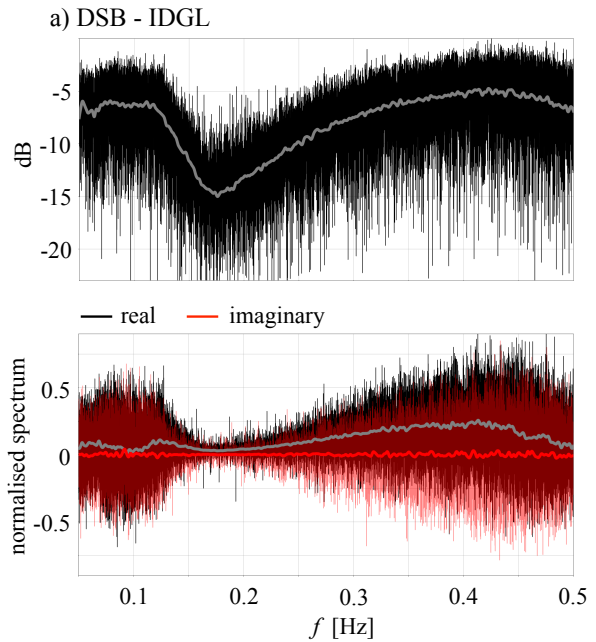

b) IDGL - IGLA
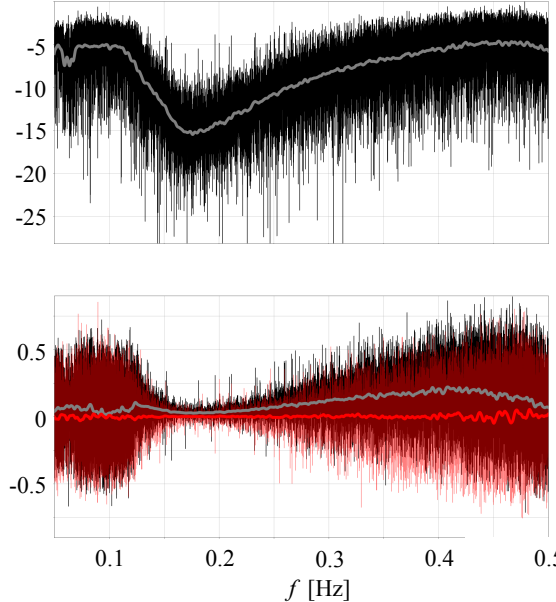

c) IGLA - ILTH
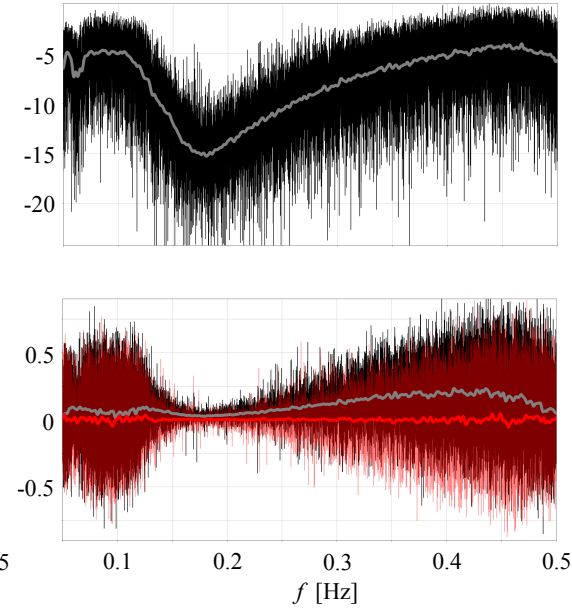

Figure 5. Frequency-domain propagation correctors $g_{i k}$ for selected receiver pairs $(i, k)$. Normalised amplitude spectra are shown above, and the separate real and imaginary parts below. As in the previous figures, smoothed versions of the spectra are shown for better visibility. Propagation correctors for other receiver pairs are very similar in character.

$n$.

Three of the 15 distinct propagation correctors (excluding auto-correlations) from the Irish Seismic Network example are shown in Fig. 5. They are generally very similar and only differ in details. All propagation correctors carry a clear imprint of the spectral whitening. Amplitudes outside the prominent secondary microseismic peak around $0.17 \mathrm{~Hz}$ are more emphasised in order to equalise spectral power across the analysed frequency band $(0.05-0.5 \mathrm{~Hz})$. The smoothed versions of the imaginary parts of the propagation correctors, shown in the lower panels of Fig. 5, are small compared to the real parts. Hence, the applied processing has only a small effect on the frequency-averaged phase, but it may have a larger effect within narrow frequency bands.

A selection of regularly and optimally processed correlations in the time and frequency domains is shown in Fig. 6, for the same station pairs as in Fig. 5. As a consequence of spectral whitening, the regularly processed correlations have a flat amplitude spectrum. This is in contrast to the optimally processed correlations, though their amplitude spectrum is still substantially flatter than the amplitude spectrum of the raw data in Fig. 3.

It follows that the processing operation cannot be perfectly factorised into a time-window- and a receiver-pair-specific component. In fact, this can be understood intuitively: For a given time window $n$, the processing has a different effect for each receiver pair $(i, k)$ because 
a) DSB - IDGL

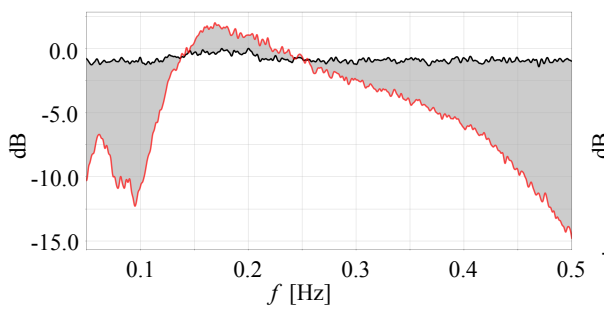

b) IDGL - IGLA

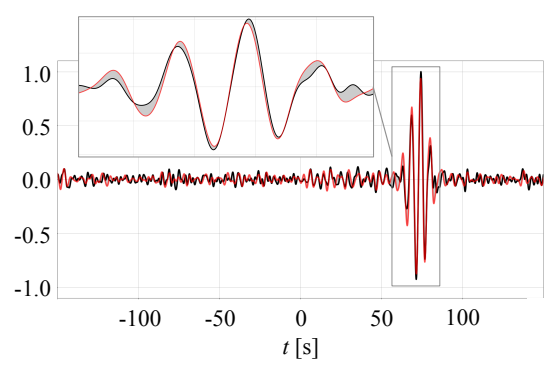

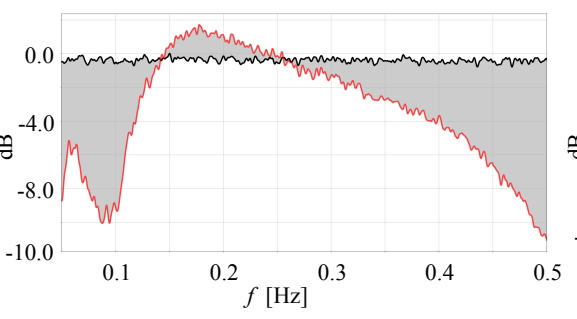

c) IGLA - ILTH
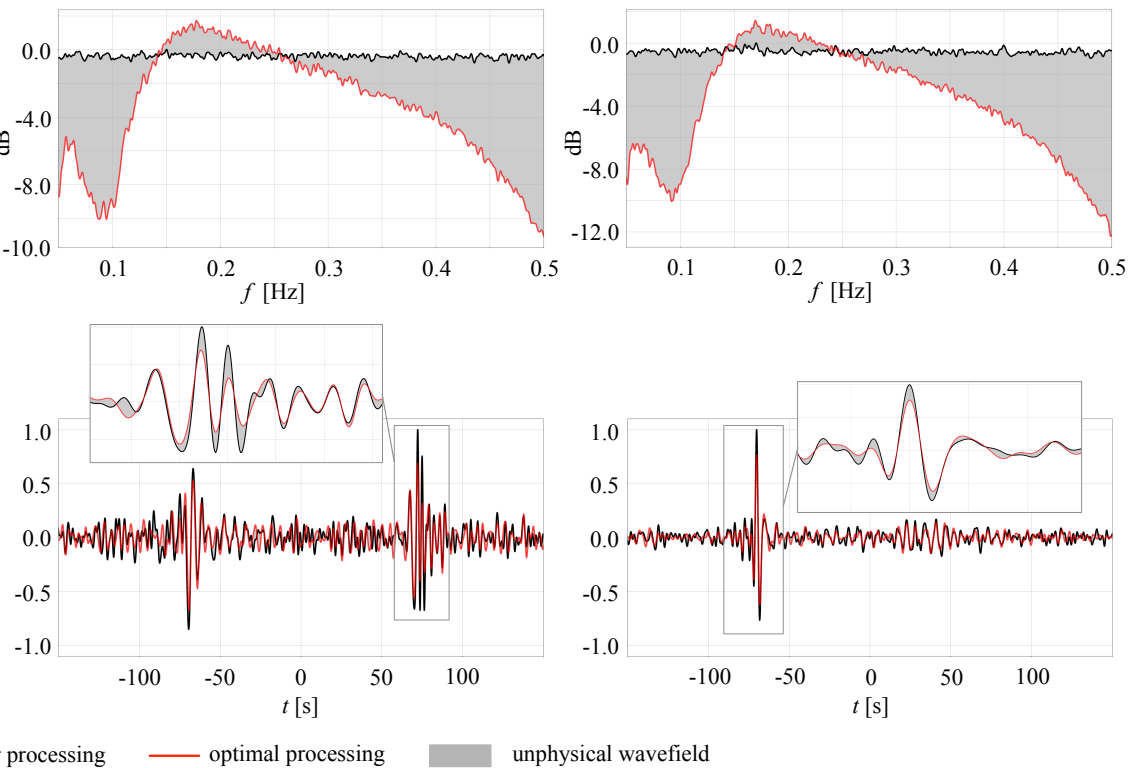

Figure 6. Amplitude spectra (top) and time-domain traces (below) of ensemble (i.e., time-averaged) correlations for the same station pairs as in Fig. 5. Regular processing is shown in black, and optimal processing in red. The estimated unphysical wavefield, shaded in grey, is the difference between the two.

Earth structure and regular wave propagation physics produce a path-specific amplitude spectrum for which the spectral whitening tries to compensate. Conversely, for a given receiver pair $(i, k)$, the processing has a different effect for each time window $n$ because the (noise) source power-spectral density changes over time. As demonstrated in Appendix A, this leads to a non-zero factorisation residual $e_{i k}^{(n)}$ and consequently to an unphysical component in the processed correlation wavefield.

This unphysical component is visualised as the grey-shaded area between the regularly and optimally processed correlations in Fig 6. It is non-zero for nearly all frequencies and reaches a maximum of around $10 \mathrm{~dB}$ in spectral amplitude for frequencies outside the secondary microseismic peak. In the time domain, the complete correlation traces are visibly affected by the unphysical component. Though regularly and optimally processed correlations resemble each other within the large-amplitude arrivals, there are local time shifts on the order of $1 \mathrm{~s}$ and amplitude variations reaching several tens of percent. Outside the large-amplitude arrivals, differences between correlation waveforms can be more substantial.

\subsection{Local scale}

We also illustrate the differences in processing using data from a local-scale array, deployed in the Lost Hills region of southern California (Clayton, 2015). The array used consists of 47 vertical-component geophones (nodes) deployed in a hexagonal shape of roughly $0.6 \mathrm{~km}$ by $0.6 \mathrm{~km}$, as shown in Fig. 7a. We use only 24 hours of recording time, specifically from 10 December 2015, and consider frequencies from $0.8 \mathrm{~Hz}$ to $4 \mathrm{~Hz}$. In this case, we find that spectral whitening and an RMS clipping as preprocessing provides reasonable waveforms, but again other choices are possible.

We follow the same procedure as described for the regional scale above. A record section emphasising the strong surface-wave energy at these frequencies can be seen in Fig. 7b. Selected examples of the difference between standard processing and the optimal version of processing are shown in Fig. 8.

Given the much larger number of station pairs, we can consider a more statistical view of these processing effects. First, we examine the travel-time bias introduced by the unphysical components of the wavefield. With a narrow-band filter between $0.8 \mathrm{~Hz}$ and $4.0 \mathrm{~Hz}$, we measure a bias in travel-time picks by cross-correlating signals in the surface-wave arrival window indicated with blue lines in Fig. 7b, assuming a wavespeed of $350 \mathrm{~m} / \mathrm{s}$. The peak of this cross-correlation indicates the timeshift for signals to be aligned, which we interpret as the bias introduced by the unphysical aspects of the processing. On average, these timeshifts represent a change of $\sim 2.9 \%$ of the total traveltimes. The geometric distribution of the traveltime biases is illustrated in Fig. 9a.

These traveltime biases are small, as expected from the overall consistency of tomographic studies based on noise correlations. However, choosing a narrower frequency band of $1-2 \mathrm{~Hz}$ for the measurements, produces average traveltime biases of $\sim 11.5 \%$, summarised in Fig. 9b. In contrast to the preavious broader-band example, this is well within the range of perturbations that one might interpret as signal in 
a) Lost Hills array

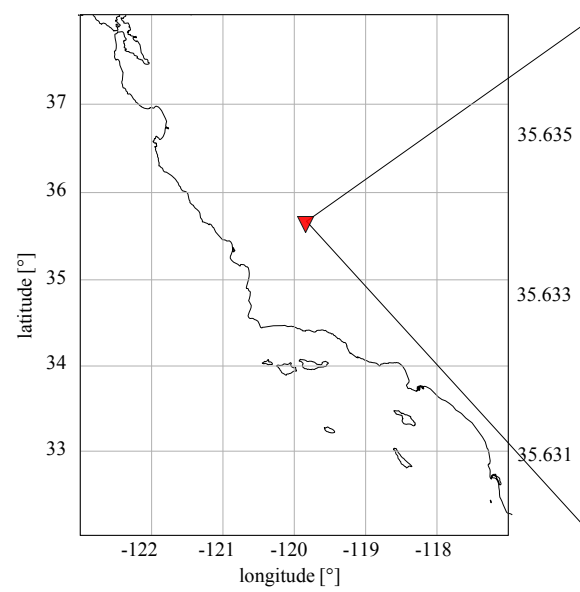

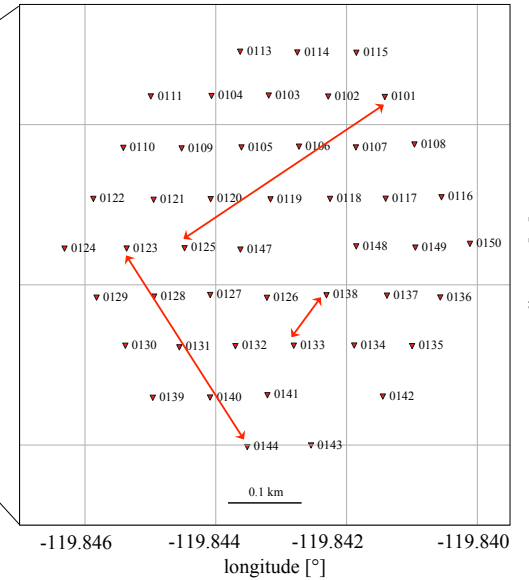

b) Record section

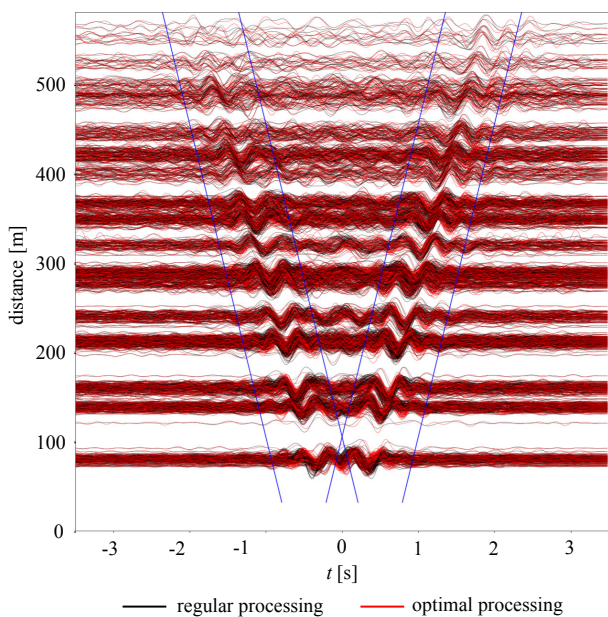

Figure 7. The Lost Hills seismic node array (Clayton, 2015). a) Geometry of the array, located north-west of Bakersfield in southern California. b) Record section showing regularly processed correlations in black (spectral whitening and rms clipping), and optimally processed correlations in red. For better visibility, traces are normalised to have equal maximum amplitudes. Examples of unnormalised traces are shown in Fig. 8 below for the station pairs indicated by red arrows in panel (a).

\section{a) $0133-0138$}
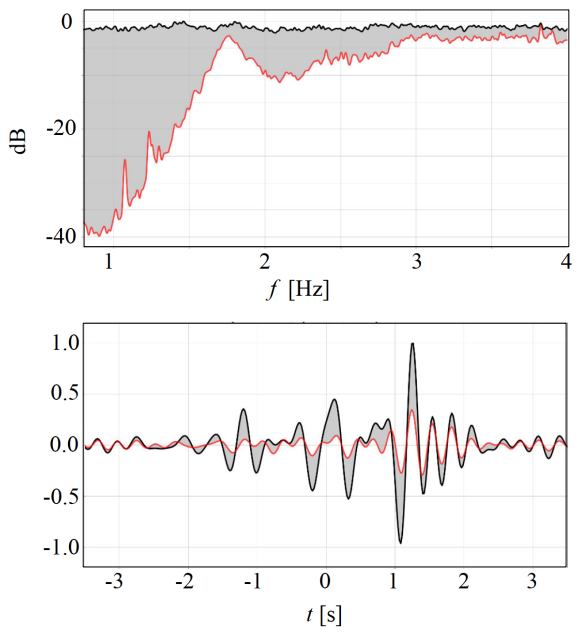

b) 0123-0144
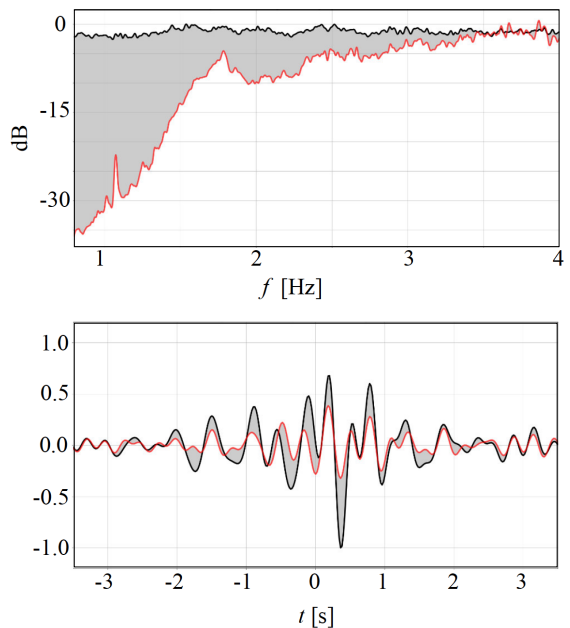

c) $0101-0125$
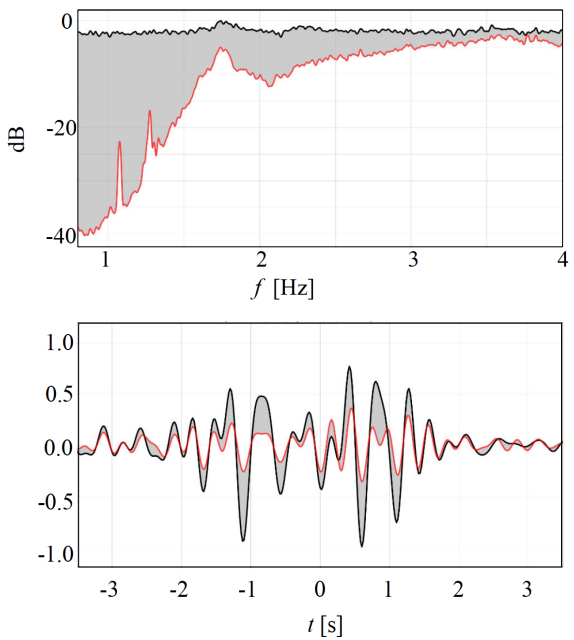

Figure 8. Amplitude spectra (top) and time domain waveforms (bottom) for noise correlations of Lost Hills station pairs 0133-0138, 0123-0144, and 01010125. Regular processing is shown in black, optimal processing in red. The difference between the two, shown in grey shading, estimates the unphysical component introduced by nonlinear processing.

temporal velocity change studies. This result suggests traveltime biases are mostly a narrow-band problem, and that they tend to average out when measurements are made over a broader frequency range. We also note from the spatial distribution of these biases in Fig. 9 that certain azimuthal patterns may be present, relating presumably to the distribution of noise sources strengthened or weakened by processing.

We may alternatively consider the implications of signal-to-noise (SNR) ratio cutoffs under different processing schemes. For our test, this ratio is defined as the maximum amplitude of a signal in the expected surface-wave arrival time window, divided by the root-mean-square of trailing signals at times after this surface-wave arrival window. If we consider the 2,162 causal and acausal signals available in the Lost Hills array, using standard spectral whitening and RMS clipping as preprocessing, we find that 92 of those traces would not meet an SNR criterion of being greater than 5 and might therefore be considered invalid. Upon applying the optimal version of that same processing, we find that actually 693 traces would not have met the same criterion. Here we see that the optimal version of processing is not as effective at bringing out a clear peak in the correlations, even though it does ensure a physically meaningful interpretation. While discarding more data might seem counter-productive, this difference implies that a significant number of traveltime measurements, in this case nearly $28 \%$ of the dataset, would have been based on signals which met our validity criterion only because of the non-physical aspects of the processing. 
a) Correlation time shifts (broader band: $0.8-4.0 \mathrm{~Hz}$ )

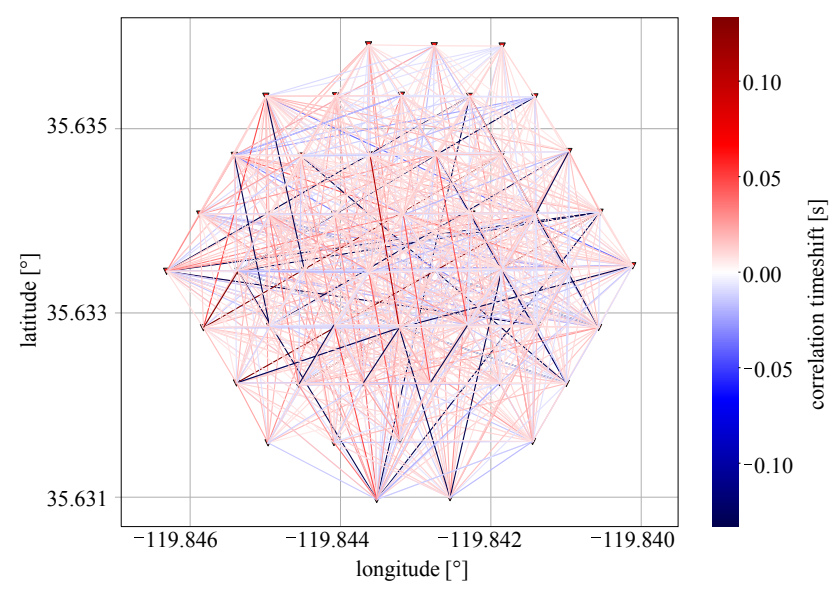

b) Correlation time shifts (narrower band: $1.0-2.0 \mathrm{~Hz}$ )

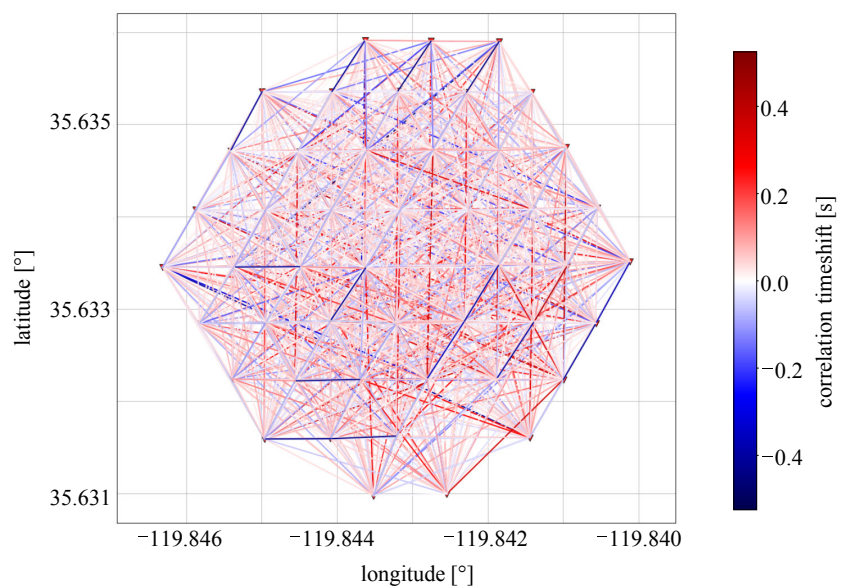

Figure 9. Unphysical surface wave traveltime biases introduced by nonlinear processing in a broader frequency band of $0.8-4.0 \mathrm{~Hz}$ (left) and a narrower frequency band of $1.0-2.0 \mathrm{~Hz}$.

\section{DISCUSSION}

We presented a datailed analysis of the effect of processing on seismic wavefield correlations, demonstrating that nonlinear processing may have undesirable effects that cannot be explained in terms of standard wave propagation physics. The positive component of this work is the derivation of optimal processing schemes, whereby unphysical effects can be completely avoided. While not being optimal in the sense of being the 'best', these processing schemes are as close as possible to some regular processing that provides useful correlations for a given dataset. In the following we discuss a priori estimates of the unphysical wavefield component, computational requirements of the optimal processing, as well as applications for which this work might be most relevant.

\subsection{Strength of the unphysical wavefield}

Based on experience with the examples presented in section 3, it is difficult to predict amplitude and phase of the unphysical wavefield component for a specific dataset and some general processing scheme. However, there are noteworthy special cases.

Most importantly, when the processing is identical for all receiver pairs $(i, k)$ and all time intervals $n$, the transfer coefficient is a constant, that is, $T_{i k}^{(n)}$. Consequently, the factorisation residual is trivially zero, and the unphysical component vanishes. This is the case, for example, when processing merely consists in the application of a bandpass filter.

Furthermore, as shown in Appendix A, the optimal factorisation is constructed such that the sum of factorisation residuals over time windows is zero, that is, $\sum_{n} e_{i k}^{(n)}=0$. It then follows from Eq. (11) that the unphysical wavefield source $\tilde{S}_{i k}$ vanishes when the power-spectral density of the sources, $S^{(n)}$ does not depend on the time inerval $n$. Hence, when the actual wavefield sources, for instance, ambient noise sources in the oceans, are stationary, the unphysical wavefield disappears, regardless of the processing scheme applied.

\subsection{Relevance}

The results in section 3.2, though being based on a specific example, suggest that the use of optimal processing schemes is important for narrow-band traveltime measurements, needed, for instance, to improve the frequency resolution of surface-wave dispersion analysis. A seemingly negative aspect of optimal processing is that more data may need to be rejected for having an insufficient signal-to-noise ratio. However, a high signal-to-noise ratio may in fact be an unphysical artifact that should be eliminated.

In addition to these general aspects, this work is likely to be most relevant in applications where correlation waveforms, amplitudes and lowamplitude arrivals, or small time shifts are exploited in order to infer Earth structure and noise sources, including their respective temporal variations.

Full-waveform ambient noise inversion is based on the direct modelling of inter-station correlations for arbitrary noise sources without the limitations of Green's function retrieval (e.g. Woodard, 1997; Tromp et al., 2010; Hanasoge, 2013; Fichtner et al., 2017; Sager et al., 2018b). Though the first real-data proof of concept of this method focused on fundamental-mode Rayleigh waves (Sager et al., 2020), future studies may in principle incorporate complete correlation waveforms. Similar to more routinely applied full-waveform inversion of event data (e.g. Igel et al., 1996; Brenders \& Pratt, 2007; Chen et al., 2007; Fichtner et al., 2009; Tape et al., 2010), this can be expected to improve tomographic resolution provided that observed and synthetic waveforms are processed carefully. Considering the examples in section 3 , this 


\section{Andreas Fichtner, Daniel Bowden \& Laura Ermert}

should include the use of optimal processing schemes, which avoid that details of correlation waveforms are mapped into artefacts.

In all examples that we considered so far, the unphysical wavefield component has a more significant effect on the amplitudes than on the phase of correlation waveforms, at least measurements are made over a broader frequency range. Though numerous applications exploited relative or scaled amplitudes across a set of observations, this still suggests that optimal processing schemes should be employed in noisecorrelation-based studies of attenuation (e.g. Cupillard \& Capdeville, 2010; Weaver, 2011; Lawrence \& Prieto, 2011; Prieto et al., 2011; Weemstra et al., 2013; Boschi et al., 2019), site amplification (e.g. Bowden et al., 2016, 2017; Viens et al., 2017), or potential earthquakeinduced ground motion (e.g. Denolle et al., 2013, 2014; Viens et al., 2017; Viens \& Denolle, 2019). Several authors have indeed applied constant transfer coefficients to raw recordings (e.g. Bowden et al., 2016, 2017) in order to extract more reliable amplitude information from noise correlations. This work provides an a posteriori justification for this approach.

Closely related to the difficulty of exploiting amplitude information is the analysis of seismic phases with amplitudes that may be small compared to a potentially unphysical component. This includes, for instance, teleseismic body waves, which are characterised by complex stationary phase regions and interference mechanisms (Forghani \& Snieder, 2010; Sager et al., 2018a) and progressively used to study deep Earth structure (e.g. Poli et al., 2012; Boué et al., 2013; Retailleau et al., 2020). With our examples in section 3 focusing on regional- and local-scale surface waves, potential unphysical effects in body waves remain to be studied.

While unphysical phase shifts induced by nonlinear processing are likely to be negligible in ambient noise tomography for static Earth structure, they may become more relevant in studies of local time-dependent subsurface variations (e.g. Brenguier et al., 2008b,a; Meier et al., 2010; Durand et al., 2011; Obermann et al., 2013, 2014; de Ridder et al., 2014; Mordret et al., 2014; Hillers et al., 2015). As mentioned in section 4.1, estimating the importance of the unphysical component a priori is difficult, except in few special cases. Yet, the machinery presented in section 2 provides a simple method to quantify the effect for a given dataset and processing scheme.

\subsection{Accounting for effective propagation and sources}

In addition to avoiding unphysical contributions through the replacement of regular by optimal processing, it is important to account for the effectiveness of the source and the wave propagation. Eq.10 is essential in this context. It demonstrates that effective wave propagation can be incorporated in the forward modelling by applying the propagation correctors $g_{i k}$ to the Green's function computed for a suitable Earth model. Alternatively, the effectiveness may be removed by deconvolving the propagation correctors from the processed correlations.

The effectiveness of the source cannot be corrected for, as it is trapped under the integral in Eq.10. However, one may infer the effective source $\tilde{S}$ by inverting processed correlations (e.g. Ermert et al., 2016, 2017; Datta et al., 2019; Xu et al., 2019; Sager et al., 2020).

\subsection{Calculation of the optimal processing}

Though the theory presented in section 2 involves the Green's function of the medium, $G$, it is not needed for the calculation of the optimal processing. In fact, the optimal processing merely involves the calculation of the factors $g_{i k}$ and $f^{(n)}$ of the transfer coefficients, which is a purely data-based operation. This is detailed in Appendix A for the scalar acoustic case, and in Appendix B for the vectorial elastic case. Thanks to the low computational cost of the factorisation, the optimal processing can be implemented as a by-product of regular processing in the frequency domain. No numerical wavefield simulations are necessary.

\section{CONCLUSIONS}

We presented a theory that quantifies the unphysical component introduced into noise correlations by nonlinear processing. It rests on a factor analysis of the discretised processing operator in the frequency domain, which tries to separate path and source effects of the processing. The factorisation residual is the contribution that cannot be separated, and it is the sole responsible for the unphysical component.

In two real-data examples we demonstrated that the unphysical component need not be small. It affects both amplitude and phase, though the former more than the latter. The unphysical phase effect is proportional to the bandwidth of the measurement in the sense that it tends to average out over frequency.

A by-product of the factor analysis are optimal processing schemes; optimal in the sense of being as close as possible to a chosen regular processing scheme, while entirely avoiding its unphysical effects. The optimal processing allows us to estimate the unphysical component, and it can be computed easily on-the-fly with regular processing, without any need for numerical simulations.

Though general predictions of the strength of processing-induced unphysical effects are difficult, unless for few special cases, we anticipate that optimal processing schemes may be most useful in applications that exploit complete correlation waveforms, amplitudes and lowamplitude arrivals, or small (time-dependent) phase shifts. 


\section{ACKNOWLEDGMENTS}

The authors would like to thank Gregor Hillers, Brian Kennett, Lise Retailleau, Korbinian Sager, and Chantal van Dinther for fruitful discussions, and Robert Clayton for kindly providing waveform data from the Lost Hills array. A research code named OPUS that implements optimal processing for example data can be found on the scientific software pages of the Seismology and Wave Physics Group at ETH Zurich (www.swp.ethz.ch).

\section{REFERENCES}

Aki, K. \& Richards, P., 2002. Quantitative Seismology, University Science Books.

Ardhuin, F., Stutzmann, E., Schimmel, M., \& Mangeney, A., 2011. Ocean wave sources of seismic noise, J. Geophys. Res., 116, doi:10.1029/2011JC006952.

Ardhuin, F., Gualtieri, L., \& Stutzmann, E., 2015. How ocean waves rock the earth: Two mechanisms explain microseisms with periods 3 to 300s, Geophys. Res. Lett., 42(3), 765-772.

Baig, A. M., Campillo, M., \& Brenguier, F., 2009. Denoising seismic noise cross correlations, J. Geophys. Res., 114, doi:10.1029/2008JB006085.

Bensen, G. D., Ritzwoller, M. H., Barmin, M. P., Levshin, A. L., Lin, F., Moschetti, M. P., Shapiro, N. M., \& Yang, Y., 2007. Processing seismic ambient noise data to obtain reliable broad-band surface wave dispersion measurements, Geophys. J. Int., 169, $1239-1260$.

Boschi, L., Magrini, F., Cammarano, F., \& van der Meijde, M., 2019. On seismic ambient noise cross-correlation and surface-wave attenuation, Geophys. J. Int., 219, 1568-1589.

Boué, P., Poli, P., Campillo, M., Pedersen, H., Briand, X., \& Roux, P., 2013. Teleseismic correlations of ambient noise for deep global imaging of the Earth, Geophys. J. Int., 194, 844-848.

Bowden, D. C., Kohler, M. D., Tsai, V. C., \& Weeraratne, D. S., 2016. Offshore Southern California lithospheric velocity structure from noise crosscorrelation functions, J. Geophys. Res., 121, 3415-3427.

Bowden, D. C., Tsai, V. C., \& Lin, F.-C., 2017. Amplification and attenuation across USArray using ambient noise wavefrom tracking, J. Geophys. Res., 122, doi:10.1002/2017JB014804.

Brenders, A. J. \& Pratt, R. G., 2007. Full waveform tomography for lithospheric imaging: results from a blind test in a realistic crustal model, Geophys. J. Int., 168, 133-151.

Brenguier, F., Campillo, M., Haziioannou, C., Shapiro, N. M., Nadeau, R. M., \& Larose, E., 2008a. Postseismic relaxation along the San Andreas fault at Parkfield from continuous seismological observations, Science, 321, 1478-1481.

Brenguier, F., Shapiro, N. M., Campillo, M., Ferrazzini, V., Duputel, Z., Coutant, O., \& Nercessian, A., 2008b. Towards forecasting volcanic eruptions using seismic noise, Nat. Geosci., 1, 126-130.

Chen, P., Zhao, L., \& Jordan, T. H., 2007. Full 3D tomography for the crustal structure of the Los Angeles region., Bull. Seismol. Soc. Am., 97, 1094-1120.

Clayton, R. W., 2015. Lost hills node arrays, doi:10.7909/ycnz-5s81, http://web.gps.caltech.edu/ clay/LostHills/LostHills.html.

Cupillard, P. \& Capdeville, Y., 2010. On the amplitude of surface waves obtained by noise correlation and the capability to recover the attenuation: a numerical approach, Geophys. J. Int., 181, 1687-1700.

Cupillard, P., Stehly, L., \& Romanowicz, B., 2011. The one-bit noise correlation: a theory based on the concepts of coherent and incoherent noise, Geophys. J. Int., 184, 1397-1414.

Curtis, A. \& Halliday, D., 2010. Directional balancing for seismic and general wavefield interferometry, Geophysics, 75, doi: 10.1190/1.3298736.

Datta, A., Hanasoge, S., \& Goudswaard, J., 2019. Finite-frequency inversion of cross-correlation amplitudes for ambient noise source directivity estimation, J. Geophys. Res., 124, 6653-6665.

de Ridder, S. A. L., Biondi, B. L., \& Clapp, R. G., 2014. Time-lapse seismic noise correlation tomography at Valhall, Geophys. Res. Lett., 41, 6116-6122.

Delaney, E., Ermert, L., Sager, K., Kritski, A., Bussat, S., \& Fichtner, A., 2017. Passive seismic monitoring with nonstationary noise sources, Geophysics, 82, KS57-KS70.

Denolle, M. A., Dunham, E. M., Prieto, G. A., \& Beroza, G. C., 2013. Ground motion prediction of realistic earthquake sources using the ambient seismic field, J. Geophys. Res., 118, doi:10.1029/2012JB009603.

Denolle, M. A., Dunham, E. M., Prieto, G. A., \& Beroza, G. C., 2014. Strong ground motion prediction using virtual earthquakes, Science, 343, 399-403.

Durand, S., Montagner, J.-P., Roux, P., Brenguier, F., Nadeau, R. M., \& Ricard, Y., 2011. Passive monitoring of anisotropy change associated with the Parkfield 2004 earthquake, Geophys. Res. Lett., 38, doi:10.1029/2011GL047875.

Ermert, L., Villasenor, A., \& Fichtner, A., 2016. Cross-correlation imaging of ambient noise sources, Geophys. J. Int., 204, 347-364.

Ermert, L., Sager, K., Afanasiev, M., Boehm, C., \& Fichtner, A., 2017. Ambient seismic source inversion in a heterogeneous Earth: Theory and application to the Earth's hum, J. Geophys. Res., 122, 9184-9207.

Fichtner, A., 2014. Source and processing effects on noise correlations, Geophys. J. Int., 197, 1527-1531. 
Fichtner, A., 2015. Source-structure trade-offs in ambient noise correlations, Geophys. J. Int., 202, 678-694.

Fichtner, A. \& Tsai, V., 2019. Theoretical foundations of noise interferometry, in Seismic Ambient Noise, pp. 109-143, Cambridge University Press, Cambridge, U.K.

Fichtner, A., Kennett, B. L. N., Igel, H., \& Bunge, H.-P., 2009. Full seismic waveform tomography for upper-mantle structure in the Australasian region using adjoint methods., Geophys. J. Int., 179, 1703-1725.

Fichtner, A., Stehly, L., Ermert, L., \& Boehm, C., 2017. Generalised interferometry - I. Theory for inter-station correlations, Geophys. J. Int., 208, 603-638.

Forghani, F. \& Snieder, R., 2010. Underestimation of body waves and feasibility of surface-wave reconstruction by seismic interferometry, The Leading Edge, 29, 790-794.

Froment, B., Campillo, M., Roux, P., Gouédard, P., Verdel, A., \& Weaver, R. L., 2010. Estimation of the effect of nonisotropically distributed energy on the apparent arrival time in correlations, Geophysics, 75, SA85-SA93.

Groos, J. C., Bussat, S., \& Ritter, J. R. R., 2012. Performance of different processing schemes in seismic noise cross-correlations, Geophys. J. Int., 188, 498-512.

Gualtieri, L., Stutzmann, E., Juretzek, C., Hadziioannou, C., \& Ardhuin, F., 2019. Global-scale analysis and modeling of primary microseisms, Geophys. J. Int., 218, 560-572.

Halliday, D. \& Curtis, A., 2008. Seismic interferometry, surface waves and source distribution, Geophys. J. Int., 175, $1067-1087$.

Hanasoge, S. M., 2013. Measurements and kernels for source-structure inversions in noise tomography, Geophys. J. Int., 192, 971-985.

Hanasoge, S. M. \& Branicki, M., 2013. Interpreting cross-correlations of one-bit filtered noise, Geophys. J. Int., 195, $1811-1830$.

Haned, A., Stutzmann, E., Schimmel, M., Kiselev, S., Davaille, A., \& Yelles-Chaouche, A., 2016. Gloal tomography using seismic hum, Geophys. J. Int., 204, 1222-1236.

Hillers, G., Husen, S., Obermann, A., Planes, T., Larose, E., \& Campillo, M., 2015. Noise-based monitoring and imaging of aseismic transient deformation induced by the 2006 Basel reservoir stimulation, Geophysics, 80, KS51-KS68.

Igel, H., Djikpesse, H., \& Tarantola, A., 1996. Waveform inversion of marine reflection seismograms for P impedance and Poisson's ratio., Geophys. J. Int., 124, 363-371.

Kennett, B. L. N., 2001. The seismic wavefield I. - Introduction and theoretical development., Cambridge University Press.

Larose, E., Derode, A., Campillo, M., \& Fink, M., 2004. Imaging from one-bit correlations of wideband diffuse wave fields, J. Appl. Phys., 95.

Lawrence, J. W. \& Prieto, G. A., 2011. Attenuation tomography in the western United States from ambient seismic noise, J. Geophys. Res., 116, doi:10.1029/2010JB007836.

Lin, F.-C., Li, D., Clayton, R. W., \& Hollis, D., 2013. High-resolution 3D shallow crustal structure in Long Beach, California: Application of ambient noise tomography on a dense seismic array, Geophysics, 78, Q45-Q56.

Lobkis, O. I. \& Weaver, R. L., 2001. On the emergence of the Green's function in the correlations of a diffuse field, J. Acoust. Soc. Am., 110, 3011-3017.

Luo, Y., Yang, Y., Xie, J., Yang, X., Ren, F., Zhao, K., \& Xu, H., 2020. Evaluating uncertainties of phase velocity measurements from cross-correlations of ambient seismic noise, Seis. Res. Lett., 91, doi:10.1785/0220190308.

McNamara, D. E. \& Boaz, R. I., 2019. Visualization of the seismic ambient noise spectrum, in Seismic Ambient Noise, pp. 1-29, Cambridge University Press, Cambridge, U.K.

Meier, U., Shapiro, N. M., \& Brenguier, F., 2010. Detecting seasonal variations in seismic velocities within the Los Angeles basin from correlations of ambient seismic noise, Geophys. J. Int., 181, 985-996.

Melo, G., Malcolm, A., Mikesell, D., \& van Wijk, K., 2013. Using SVD for improved interferometric Green's function retrieval, Geophys. J. Int., 1596-1612.

Menon, R., Gerstoft, P., \& Hodgkiss, W. S., 2012. Cross-correlations of diffuse noise in an ocean environment using eigenvalue based statistical inference, J. Acoust. Soc. Am., 132, 3213-3224.

Mordret, A., Shapiro, N., \& Singh, S., 2014. Seismic noise-based time-lapse monitoring of the Valhall overburden, Geophys. Res. Lett., 41, 49454952.

Mulargia, F., 2012. The seismic noise wavefield is not diffuse, J. Acoust. Soc. Am., 131, 2853-2858.

Nakata, N. \& Nishida, K., 2019. Body wave exploration, in Seismic Ambient Noise, pp. 218-238, Cambridge University Press, Cambridge, U.K.

Nakata, N., Chang, J. P., Lawrence, J. F., \& Boué, P., 2015. Body wave extraction and tomography at Long Beach, California, with ambient-noise interferometry, J. Geophys. Res., 120, 1159-1173.

Obermann, A., T.Planes, Larose, E., \& Campillo, M., 2013. Imaging preeruptive and coeruptive structural and mechanical changes of a volcano with ambient seismic noise, J. Geophys. Res., 118, 1-10.

Obermann, A., Froment, B., Campillo, M., Larose, E., Planes, T., Valette, B., Chen, J.-H., \& Liu, Q. Y., 2014. Seismic noise correlations to image structural and mechanical changes associated with the Mw 7.9 2008 Wenchuan earthquake, J. Geophys. Res., 119, doi:10.1002/2013JB010932.

Peterson, J., 1993. Observations and modeling of seismic background noise, USGS Open File Report, 93-322, 94 pp.

Poli, P., Campillo, M., Pedersen, H., \& LAPNET Working Group, 2012. Body-wave imaging of Earth's mantle discontinuities from ambient 
seismic noise, Science, 38, 1063-1065.

Prieto, G. A., Denolle, M., Lawrence, J. F., \& Beroza, G. C., 2011. On amplitude information carried by the ambient seismic field, $C$. $R$. Geoscience, 343, 600-614.

Retailleau, L. \& Gualtieri, L., 2019. Toward high-resolution period-dependent seismic monitoring of tropical cyclones, Geophys. Res. Lett., 46, 13291337.

Retailleau, L., Boué, P., Li, L., \& Campillo, M., 2020. Ambient seismic noise imaging of the lowermost mantle beneath the North Atlantic Ocean, Geophys. J. Int., in press, doi:10.1093/gji/gga210.

Ritzwoller, M. H. \& Feng, L., 2019. Overview of pre- and post-processing of ambient noise correlations, in Seismic Ambient Noise, pp. 144-187, Cambridge University Press, Cambridge, U.K.

Sager, K., Boehm, C., Ermert, L., Krischer, L., \& Fichtner, A., 2018a. Sensitivity of seismic noise correlation functions to global noise sources, J. Geophys. Res., 123, 6911-6921.

Sager, K., Ermert, L., Boehm, C., \& Fichtner, A., 2018b. Towards full waveform ambient noise inversion, Geophys. J. Int., 212, 566-590.

Sager, K., Boehm, C., Ermert, L., Krischer, L., \& Fichtner, A., 2020. Global-scale full-waveform ambient noise inversion, J. Geophys. Res., 125.

Sánchez-Sesma, F. J. \& Campillo, M., 2006. Retrieval of the Green's function from cross correlation: The canonical elastic problem, Bull. Seis. Soc. Am., 96, 1182-1191.

Saygin, E. \& Kennett, B. L. N., 2012. Crustal structure of Australia from ambient seismic noise tomography, J. Geophys. Res., 117, doi:10.1029/2011JB008403.

Schimmel, M. \& Paulssen, H., 1997. Noise reduction and detection of weak, coherent signals through phase-weighted stacks, Geophys. J. Int., 130, 497-505.

Schimmel, M., Stutzmann, E., \& Gallart, J., 2011. Using instantaneous phase coherence for signal extraction from ambient noise data at a local to a global scale, Geophys. J. Int., 184, 494-506.

Seats, K. J., Lawrence, J. F., \& Prieto, G. A., 2012. Improved ambient noise correlation functions using Welch's method, Geophys. J. Int., 188, 513-523.

Seydoux, L., de Rosny, J., \& Shapiro, N. M., 2017. Pre-processing ambient noise cross-correlations with equalizing the covariance eigenspectrum, Geophys. J. Int., 210, 1432-1449.

Shapiro, N. M., 2019. Applications with surface waves extracted from ambient seismic noise, in Seismic Ambient Noise, pp. 218-238, Cambridge University Press, Cambridge, U.K.

Shapiro, N. M. \& Campillo, M., 2004. Emergence of broadband Rayleigh waves from correlations of the ambient seismic noise, Geophys. Res. Lett., 31, doi:10.1029/2004GL019491.

Shapiro, N. M., Campillo, M., Stehly, L., \& Ritzwoller, M., 2005. High resolution surface wave tomography from ambient seismic noise, Science, 307, 1615-1618.

Shen, Y., Ren, Y., Gao, H., \& Savage, B., 2012. An improved method to extract very-broadband empirical Green's functions from ambient seismic noise, Bull. Seis. Soc. Am., 102, 1872-1877.

Snieder, R. \& Şafak, E., 2006. Extracting the building response using seismic interferometry: Theory and application to the Millikan Library in Pasadena, California, Bull. Seis. Soc. Am., 96, 586-598.

Snieder, R., Miyazawa, M., Slob, E., Vasconcelos, I., \& Wapenaar, K., 2009. A comparison of strategies for seismic interferomtry, Surv. Geophys., 30, 503-523.

Stehly, L., Campillo, M., \& Shapiro, N. M., 2006. A study of the seismic noise from its longrange correlation properties, J. Geophys. Res., 111, doi:10.1029/2005JB004237.

Stehly, L., Fry, B., Campillo, M., Shapiro, N. M., Guilbert, J., Boschi, L., \& Giardini, D., 2009. Tomography of the Alpine region from observations of seismic ambient noise, Geophys. J. Int., 178, 338-350.

Stehly, L., Cupillard, P., \& Romanowicz, B., 2011. Towards improving ambient noise tomography using simultaneously curvelet denoising filters and SEM simulations of seismic ambient noise, Com. Rend. Geosc., 343, 591-599.

Stutzmann, E., Ardhuin, F., Schimmel, M., Mangeney, A., \& Patau, G., 2012. Modelling long-term seismic noise in various environments, Geophys. J. Int., 191, 707-722.

Tape, C., Liu, Q., Maggi, A., \& Tromp, J., 2010. Seismic tomography of the southern California crust based upon spectral-element and adjoint methods., Geophys. J. Int., 180, 433-462.

Tian, Y. \& Ritzwoller, M. H., 2015. Directionality of ambient noise on the Juan de Fuca plate: implications for source locations of the primary and secondary microseisms, Geophys. J. Int., 201(1), 429-443.

Tromp, J., Luo, Y., Hanasoge, S., \& Peter, D., 2010. Noise cross-correlation sensitivity kernels, Geophys. J. Int., 183, 791-819.

Tsai, V. C., 2009. On establishing the accuracy of noise tomography traveltime measurements in a realistic medium, Geophys. J. Int., 178, $1555-1564$.

Tsai, V. C., 2011. Understanding the amplitudes of noise correlation measurements, J. Geophys. Res., 116, doi:10.1029/2011JB008483.

van Dalen, K. N., Wapenaar, K., \& Halliday, D. F., 2014. Surface wave retrieval in layered media using seismic interferometry by multidimensional deconvolution, Geophys. J. Int., 196, 230-242.

Vasconcelos, I. \& Snieder, R., 2008a. Interferometry by deconvolution, Part 1 - Theory for acoustic waves and numerical examples, 
Geophysics, 73, S129-S141.

Vasconcelos, I. \& Snieder, R., 2008b. Interferometry by deconvolution, Part 2 - Theory for elastic waves and application to drill-bit seismic imaging, Geophysics, 73, S115-S128.

Viens, L. \& Denolle, M. A., 2019. Long-period ground motions from past and virtual megathrust earthquakes along the Nankai Trough, Japan, Bull. Seis. Soc. Am., 109, 1312-1330.

Viens, L., Denolle, M. A., Miyake, H., Sakai, S., \& Nakagawa, S., 2017. Retrieving impulse response function amplitudes from the ambient seismic field, Geophys. J. Int., 210, 210-222.

Wapenaar, K., 2004. Retrieving the elastodynamic Green's function of an arbitrary inhomogeneous medium by cross correlation, Phys. Rev. Lett., 93, 254301.

Wapenaar, K. \& Fokkema, J., 2006. Green's function representations for seismic interferometry, Geophysics, 71, SI33-SI46.

Wapenaar, K. \& van der Neut, J., 2010. A representation for Greens function retrieval by multidimensional deconvolution, J. Acoust. Soc. Am., 128, 366-371.

Wapenaar, K., Ruigrok, E., van der Neut, J., \& Draganov, D., 2011a. Improved surface-wave retrieval from ambient seismic noise by multi-dimensional deconvolution, Geophys. Res. Lett., 38, doi:10.1029/2010GL045523.

Wapenaar, K., van der Neut, J., Ruigrok, E., Draganov, D., Hunziker, J., Slob, E., Thorbecke, J., \& Snieder, R., 2011b. Seismic interferometry by crosscorrelation and by multidimensional deconvolution: a systematic comparison, Geophys. J. Int., 185, 1335-1364.

Weaver, R. L., 2008. Ward identities and the retrieval of Green's functions in the correlations of a diffuse field, Wave Motion, 45, 596-604. Weaver, R. L., 2011. On the amplitudes of correlations and the inference of attenuations, specific intensities and site factors from ambient noise, C. R. Geoscience, 343, 615-622.

Weaver, R. L. \& Lobkis, O. I., 2004. Diffuse fields in open systems and the emergence of Green's function, J. Acoust. Soc. Am., 116, 2731-2734.

Weaver, R. L. \& Yoritomo, J., 2018. Temporally weighting a time varying noise field to improve Green function retrieval, J. Acoust. Soc. Am., 143, 3706-3719.

Weemstra, C., Boschi, L., Goertz, A., \& Artman, B., 2013. Seismic attenuation from recordings of ambient noise, Geophysics, 78, Q1-Q14. Welch, P. D., 1967. The use of fast Fourier transform for the estimation of of power spectra: A method based on time-averaging over short, modified periodograms, IEEE Trans. Audio Electroacoust., 15, 70-73.

Woodard, M. F., 1997. Implications of localized, acoustic absorption for heliotomographic analysis of sunspots, Astrophys. J., 485, $890-894$.

Xu, Z., Mikesell, T. D., Gribler, G., \& Mordret, A., 2019. Rayleigh-wave multicomponent cross-correlation-based source inversion. Part 1: Theory and numerical examples, Geophys. J. Int., 218, 1761-1780.

Yao, H. \& van der Hilst, R. D., 2009. Analysis of ambient noise energy distribution and phase velocity bias in ambient noise tomography, with application to SE Tibet, Geophys. J. Int., 179, 1113-1132.

Yoritomo, J. Y. \& Weaver, R. L., 2016. Fluctuations in the cross-correlation for fields lacking full diffusivity: The statistics of spurious features, J. Acoust. Soc. Am., 140, 702-713.

Zhan, Z., Tsai, V. C., \& Clayton, R. W., 2013. Spurious velocity changes caused by temporal variations in ambient noise frequency content, Geophys. J. Int., 194, 1574-1581.

\section{APPENDIX A: OPTIMAL FACTORISATION OF THE SCALAR TRANSFER COEFFICIENT (ACOUSTIC CASE)}

The minimisation of the unphysical interferometric wavefield requires the minimisation of the unphysical wavefield source $\tilde{S}_{i k}\left(\mathbf{x}, \mathbf{x}^{\prime}\right)$, which, according to Eq. (11), is the average of individual power-spectral densities $S^{(n)}\left(\mathbf{x}, \mathbf{x}^{\prime}\right)$ weighted by the factorisation residuals $e_{i k}^{(n)}$. Hence, the optimal $e_{i k}^{(n)}$ that minimise $\tilde{S}_{i k}\left(\mathbf{x}, \mathbf{x}^{\prime}\right)$ depend on the time- and space-dependent power-spectral densities $S^{(n)}\left(\mathbf{x}, \mathbf{x}^{\prime}\right)$. Though these are generally unknown, it is meaningful to request that $\tilde{S}_{i k}\left(\mathbf{x}, \mathbf{x}^{\prime}\right)$ be zero in the special case where the individual sources $S^{(n)}\left(\mathbf{x}, \mathbf{x}^{\prime}\right)$ are independent of the window index $n$. It then follows from Eq. (11) that the factorisation residuals must average to zero over time, that is,

$e_{i k}=\frac{1}{N} \sum_{n=1}^{N} e_{i k}^{(n)}=0$.

Hence, the presence of any unphysical wavefield is ultimately caused by the time variability of the individual sources $S^{(n)}\left(\mathbf{x}, \mathbf{x}^{\prime}\right)$. When ambient field sources are stationary over time, any processing will not induce an unphysical wavefield.

The factorisation of the transfer coefficient in (8) is inherently non-unique because the source corrector $f^{(n)}$ and the propagation corrector $g_{i k}$ trade off by an arbitrary, complex-valued factor. To remove this ambiguity, we enforce the additional constraint that the source correctors average to 1 , meaning that

$\frac{1}{N} \sum_{n=1}^{N} f^{(n)}=1$. 
The source correctors act as relative weights of the time-dependent sources $S^{(n)}\left(\mathbf{x}, \mathbf{x}^{\prime}\right)$, and so (A2) ensures that the average weight is unity. Now summing (8) over $n$ and using (A1) and (A2), determines the propagation correctors as time averages of the transfer coefficients,

$g_{i k}=\frac{1}{N} \sum_{n=1}^{N} T_{i k}^{(n)}$.

To obtain the source correctors $f^{(n)}$, we consider the squared norm of the factorisation residuals $e_{i k}^{(n)}$ for a fixed $n$,

$\sum_{i, k}\left(e_{i k}^{(n)}\right)^{2}=\sum_{i, k}\left(T_{i k}^{n}-f^{(n)} g_{i k}\right)\left(T_{i k}^{n}-f^{(n)} g_{i k}\right)^{*}$.

Forcing the derivative of (A4) with respect to $f^{(n)}$ to zero, we find

$\operatorname{Re} f^{(n)}=\frac{\operatorname{Re} \sum_{i, k} T_{i k}^{(n)} g_{i k}^{*}}{\sum_{i, k}\left|g_{i k}\right|^{2}}$.

Eq. (A5) only determines the real part of $f^{(n)}$, leaving us the freedom to set $\operatorname{Im} f^{(n)}=0$. This choice ensures that the phase of the effective power-spectral density $\tilde{S}\left(\mathbf{x}, \mathbf{x}^{\prime}\right)$ is zero for $\mathbf{x}=\mathbf{x}^{\prime}$.

\section{APPENDIX B: EXTENSION TO ELASTIC WAVE PROPAGATION}

For the elastic case we first note that the vectorial equivalent of the forward modelling equation (4) is given by

$\mathbf{I}\left(\mathbf{x}_{i}, \mathbf{x}_{k}\right)=\int \mathbf{G}\left(\mathbf{x}_{i}, \mathbf{x}\right)\left[\int \mathbf{S}\left(\mathbf{x}, \mathbf{x}^{\prime}\right) \mathbf{G}^{*}\left(\mathbf{x}^{\prime}, \mathbf{x}_{k}\right) d \mathbf{x}^{\prime}\right] d \mathbf{x}$

with the tensorial interferogram $\mathbf{I}$, the Green's tensor $\mathbf{G}$, and the tensorial power-spectral density $\mathbf{S}$. We neglected noise sensu stricto in the interest of readability. The corresponding relation between processed and unprocessed interferograms is

$\tilde{\mathbf{I}}^{(n)}\left(\mathbf{x}_{i}, \mathbf{x}_{k}\right)=\mathbf{T}_{i k}^{(n)} \mathbf{I}^{(n)}\left(\mathbf{x}_{i}, \mathbf{x}_{k}\right)$,

where $\mathbf{T}_{i k}^{(n)}$ is the tensorial transfer coefficient. In analogy to Eq. (8), we introduce the factorisation

$\mathbf{T}_{i k}^{(n)}=\mathbf{g}_{i k} f^{(n)}+\mathbf{e}_{i k}^{(n)}$,

involving the propagation corrector $\mathbf{g}_{i k}$, the source corrector $f^{(n)}$, and the factorisation residual $\mathbf{e}_{i k}^{(n)}$. In terms of these, processed interferograms can be computed as

$\tilde{\mathbf{I}}\left(\mathbf{x}_{i}, \mathbf{x}_{k}\right)=\int \mathbf{g}_{i k} \mathbf{G}\left(\mathbf{x}_{i}, \mathbf{x}\right)\left[\int \tilde{\mathbf{S}}\left(\mathbf{x}, \mathbf{x}^{\prime}\right) \mathbf{G}^{*}\left(\mathbf{x}^{\prime}, \mathbf{x}_{k}\right) d \mathbf{x}^{\prime}\right] d \mathbf{x}+\mathbf{E}\left(\mathbf{x}_{i}, \mathbf{x}_{k}\right)$

with the effective source

$\tilde{\mathbf{S}}\left(\mathbf{x}, \mathbf{x}^{\prime}\right)=\frac{1}{N} \sum_{n=1}^{N} f^{(n)} \mathbf{S}^{(n)}\left(\mathbf{x}, \mathbf{x}^{\prime}\right)$,

and the unphysical component $\mathbf{E}\left(\mathbf{x}_{i}, \mathbf{x}_{k}\right)$. To derive the optimal factorisation, we follow the same approach as in Appendix A. To condense notation, we denote the scalar product between two matrices $\mathbf{A}$ and $\mathbf{B}$ as $\mathbf{A}: \mathbf{B}=\sum_{i, k=1}^{3} A_{i k} B_{i k}$. First, we request that the time-averaged factorisation residuals vanish component-wise for each receiver pair,

$\frac{1}{N} \sum_{n=1}^{N} \mathbf{e}_{i k}^{(n)}=\mathbf{0}$,

and that the time-averaged source correctors are equal to 1 ,

$\frac{1}{N} \sum_{n=1}^{N} f^{(n)}=1$.

Averaging (B3) over all time intervals then yields the tensorial propagation corrector

$\mathbf{T}_{i k}=\frac{1}{N} \sum_{n=1}^{N} \mathbf{T}_{i k}^{(n)}=\mathbf{g}_{i k}$.

To minimise the factorisation residuals $\mathbf{e}_{i k}^{(n)}$ per time window, we consider the $L_{2}$-norm

$L^{(n)}=\sum_{i, k}\left|\mathbf{e}_{i k}^{(n)}\right|^{2}=\sum_{i, k} \mathbf{e}_{i k}^{(n)}: \mathbf{e}_{i k}^{(n) *}=\left[\mathbf{T}_{i k}^{(n)}-f^{(n)} \mathbf{g}_{i k}\right]:\left[\mathbf{T}_{i k}^{(n)}-f^{(n)} \mathbf{g}_{i k}\right]^{*}$.

Setting the derivative of (B9) with respect to $f^{(n)}$ to zero, gives

$0=\operatorname{Re} f^{(n)} \sum_{i, k}\left|\mathbf{g}_{i k}\right|^{2}-\operatorname{Re} \sum_{i, k} \mathbf{T}_{i k}^{(n)}: \mathbf{g}_{i k}^{*}$ 
From (B10) we finally obtain

$f^{(n)}=\frac{\operatorname{Re} \sum_{i, k} \mathbf{T}_{i k}^{(n)}: \mathbf{g}_{i k}^{*}}{\sum_{i, k}\left|\mathbf{g}_{i k}\right|^{2}}$

As in the scalar case, the imaginary part of the source correctors is unconstrained. The physically most meaningful solution is again to require the imaginary part of $f^{(n)}$ to vanish, which ensures that the phase of the original power-spectral density $\mathbf{S}$ equals the phase of the effective power-spectral density $\tilde{\mathbf{S}}$. 